\title{
Thermodynamics conditions of matter in neutron star mergers
}

\author{
Albino Perego ${ }^{1,2}$, Sebastiano Bernuzzi ${ }^{3}$, and David Radice ${ }^{4,5}$ \\ 1 Department of Physics, Trento University, Via Sommarive 14, 38123 Trento, Italy \\ 2 Istituto Nazionale di Fisica Nucleare, Sezione di Milano-Bicocca, Piazza della Scienza 20100, Milano, Italy \\ 3 Theoretisch-Physikalisches Institut, Friedrich-Schiller-Universität Jena, 07743, Jena, German \\ 4 Institute for Advanced Study, 1 Einstein Drive, Princeton, NJ 08540, USA \\ 5 Department of Astrophysical Sciences, Princeton University, 4 Ivy Lane, Princeton, NJ 08544, USA
}

Received: date / Revised version: date

\begin{abstract}
Matter in neutron star collisions can reach densities up to few times the nuclear saturation threshold $\left(\rho_{0} \simeq 2.7 \times 10^{14} \mathrm{~g} \mathrm{~cm}^{-3}\right.$ ) and temperatures up to one hundred $\mathrm{MeV}$. Understanding the structure and composition of such matter requires many-body nonperturbative calculations that are currently highly uncertain. Unique constraints on the neutron star matter are provided by gravitational-wave observations aided by numerical relativity simulations. In this work, we explore the thermodynamical conditions of matter and radiation along the merger dynamics. We consider 3 microphysical equation of state models and numerical relativity simulations including an approximate neutrino transport scheme. The neutron star cores collision and their multiple centrifugal bounces heat the initially cold matter to several tens of $\mathrm{MeV}$. Streams of hot matter with initial densities $\sim 1-2 \rho_{0}$ move outwards and cool due to decompression and neutrino emission. The merger can result in a neutron star remnant with densities up to $3-5 \rho_{0}$ and temperatures $\sim 50 \mathrm{MeV}$. The highest temperatures are confined in an approximately spherical annulus at densities $\sim \rho_{0}$. Such temperatures favour positron-neutron capture at densities $\sim \rho_{0}$, thus leading to a neutrino emission dominated by electron antineutrinos. We study the impact of trapped neutrinos on the remnant matter's pressure, electron fraction and temperature and find that it has a negligible effect. Disks around neutron star or black hole remnant are neutron rich and not isentropic, but they differ in size, entropy and lepton fraction depending on the nature of the central object. In presence of a black hole, disks are smaller and mostly transparent to neutrinos; in presence of a massive neutron star, they are more massive, geometrically and optically thick.
\end{abstract}

PACS. 04.25.D numerical relativity - 04.30.Db gravitational wave generation and sources - 95.30.Sf relativity and gravitation - 95.30.Lz Hydrodynamics - 97.60.Jd Neutron stars

\section{Introduction}

Simulation in numerical relativity (NR) predict that the outcome of binary neutron star (BNS) mergers on dynamical timescales is the prompt formation of a black hole via gravitational collapse or of a remnant neutron star (NS) 11. In the latter case, the cold, $\beta$-equilibrated matter of the individual NSs at typical densities 11 of $\rho_{\mathrm{NS}} \sim 2-3 \rho_{0}$ is pushed to $\rho \sim 5-6 \rho_{0}$ and temperatures up to $T \sim$ $50-100 \mathrm{MeV}$ in the remnant (see e.g. 2, 3,4]). Those extreme conditions are reached on a timescale of milliseconds, and potentially differ from those of proto NS in supernova remnants (e.g. [5]).

Our knowledge of the properties of cold matter at supranuclear densities are still affected by large uncertainties, see e.g. 6, 7]. Nevertheless they are key in determin-

Send offprint requests to: albino.perego@unitn.it

1 We consider here NSs of "fiducial" mass of $M_{\mathrm{NS}} \sim 1.35-$ $1.4 M_{\odot}$. ing the internal structure and mass-radius relation of NSs. Despite many theoretical effords, also the behavior of nuclear matter at finite temperature is poorly known, see e.g. [8] and references therein. The first composition- and temperature-dependent versions of the equation of state (EOS) widely used in simulations of binary NS mergers and core-collapse supernovae were the Lattimer-Swesty (LS) EOS 9 and the Shen EOS [10. Most of the EOS currently used and compatible with present nuclear constraints are based on the relativistic mean field (RMF) approach [8. However, more realistic EOSs based on detailed microscopic interactions and many-body techniques are becoming available, e.g. [11.

A detailed knowledge of the matter interactions in the merger remnant is pivotal for characterizing the dynamics and the related gravitational and electromagnetic radiation signatures. For example, tidal interactions during the inspiral are parametrized by NS compactness and EOSdependent tidal polarizability coefficients $12,13,14,15,16$, 17]; the properties of the merger dynamics dependent pri- 
marily on those coefficients 18,19. Moreover, the maximum mass of nonrotating NS supported by the EOS determines the threshold for the prompt gravitational collapse of the remnant 20,21. Similarly, the EOS characterizes the remnant NS, the disk, the ejecta, and the neutrino emission properties (e.g. 222,4]), thus influencing the nucleosynthesis and ultimately the properties of the kilonova emission 4,23. The timescale for the gravitational collapse to a black hole, as well as the mass and the lifetime of the disk, can also play a pivotal role in launching a relativistic jet and producing a (short) gamma-ray burst, see e.g. [24,25],26,27]. The appearance of phase transitions and extra degrees of freedom, including hyperons and quarks, can soften the EOS in the merger remnant leading to collapse and/or signatures in the gravitational waves (GWs) signal [2,28, 29, 30].

NR simulations are the only means to build models and to interpret the observations that set constraints on the NS properties and thus on the EOS. The measurement of tidal polarizability parameters in the GW170817 signal constrained the EOS at $\rho \lesssim \rho_{\mathrm{NS}}$, e.g. 31,32,33,34. NR simulations support the development of tidal waveform models for such measurement, e.g. 35, 17, 36, 37,38. Similarly, the post-merger signal from the remnant, although not detected in GW170817 39, could also place strong constraints on the physics at extreme densities $\rho \lesssim 5-6 \rho_{0}$ 40, 41, 42, 28, 43, 44.

Simulations results combined with the ejecta properties inferred from AT2017gfo (the kilonova associated with GW170817) led to upper bounds on the maximum mass of NS by noticing that the energy of the ejecta inferred from optical and infrared data are likely incompatible with both a prompt collapse and a long lived remnant 45] (see also [46, 47,48]). Similar arguments, combined with empirical relations between NS radii and the threshold mass for prompt collapse mentioned above, tentatively rule out EOSs predicting very small NS radii 49 . The correlation between the disk mass and the tidal polarizability parameter observed in NR simulations can complement the constraints on the tidal coefficients in joint observation of GW and electromagnetic (EM) radiation [22,50].

In this work we study in details the thermodynamical conditions of matter and radiation during binary NS (BNS) mergers. We explore three microphysical EOS models by simulating the matter flow along the general relativistic merger dynamics and including weak interactions. Our work aims at quantitatively investigating the typical densities, temperatures, and electron fractions that can be reached in real astrophysical mergers and their intrinsic variability. We also explore the properties and the potential role of a gas of dynamically trapped neutrinos deep inside the merger remnant. We finally point out the robust features and some of the main uncertainties of current state-of-art simulations.

The paper is structured as follows: in Section 2 we present the EOSs employed in this work, the NR simulations setups, and the method used for the analysis of the neutrino trapped components. Section 3 is devoted to the presentation of the thermodynamical conditions expe- rienced by the astrophysical plasma as obtained by several BNS merger models, characterized by different EOSs, NS masses and physical ingredients (e.g. turbulent viscosity). In Section 4 we report the results of the analysis of the role of trapped neutrinos, while in Section 5 we discuss the properties of disks in BNS merger remnants. Finally, we conclude in Section 6

\section{Method}

We consider three different nuclear EOS models: HS(DD2) [53,54, LS220 [9], and HS(SFHo) [55], see Tab. 11 (in the following, we will refer to the first and third ones simply as DD2 and SFHo, respectively). The LS220 EOS is based on a liquid droplet Skyrme model with a value of $220 \mathrm{MeV}$ for the modulus of the nuclear incompressibility. It includes surface effects and models $\alpha$-particles as an ideal, classical, non-relativistic gas. Heavy nuclei are treated using the so-called single nucleus approximation (SNA). The DD2 and SFHo EOSs combine a statistical ensemble of several thousands of nuclei, under the assumption of nuclear statistical equilibrium (NSE), with a RMF approach for the unbound nucleons to treat high-density nuclear matter [54]. The phase transition from nuclei to homogeneous nuclear matter at densities close to nuclear saturation density is achieved by an excluded volume mechanism. DD2 and SFHo use different parameterizations and values for modeling the mean-field nuclear interactions. In particular, DD2 uses a linear, but density dependent coupling [53], while the RMF parametrization of SFHo is motivated by neutron star radius measurements from low-mass X-ray binaries (55] and references therein).

All three models have symmetry energies at saturation density within experimental bounds. LS220 has a significantly steeper density dependence of its symmetry energy than the other models. In all models, the finite temperature behavior of the EOS is mainly determined by the nucleon effective mass, $m_{N}^{*}$, with smaller effective masses leading to higher temperatures for constant entropy. The LS220 EOS assumes that the nucleon mass is the bare nucleon mass at all densities, while SFHo has $m_{N}^{*} / m_{N}=$ 0.76 at saturation density, and DD2 has $m_{N}^{*} / m_{N}=0.56$, where $m_{N}$ is the bare nucleon mass.

These EOSs predict NS maximum masses and radii within the range allowed by current astrophysical constraints, including the recent LIGO-Virgo constraint on tidal deformability $31,32,34,33$. SFHo, LS220, and DD2 support $2.06,2.06$, and $2.42 M_{\odot}$ cold, non-rotating maximum NS masses. The cold, non-rotating NS radius corresponding to $1.4 M_{\odot}$ is $R_{1.4}=11.9,12.7,13.2 \mathrm{~km}$ respectively. Since NS radii correlate with the pressure at roughly twice saturation density [6], we refer to EOS having smaller $R_{1.4}$ as being "softer" and to EOS having larger $R_{1.4}$ as being stiffer. In Tab. 1 we summarize the main nuclear properties of the cold EOS models used in this work.

For each EOS model, irrotational BNS configurations in quasi-circular orbit are computed by solving the general relativistic initial data problem. We use the Lorene 
Table 1. Summary of the hadronic equation of state models considered in this work, with their nuclear matter properties at saturation density $\left(n_{0}\right)$ and zero temperature: binding energy $\left(E_{0}\right)$, incompressibility $(K)$, symmetry energy $(S)$, slope of the symmetry energy $(L)$, radius of a $1.4 M_{\odot}$ NS $\left(R_{1.4}\right)$, maximum cold, irrotational NS mass $\left(M_{\max }\right)$. Experimental and observational results were taken from the comprehensive review of 8 ] and from $51,52,50$.

\begin{tabular}{|c|c|c|c|c|c|c|c|c|c|}
\hline$\overline{\mathrm{EOS}}$ & Brief description & $\begin{array}{c}n_{0} \\
{[\mathrm{fm}]^{-3}}\end{array}$ & $\begin{array}{c}E_{0} \\
{[\mathrm{MeV}]}\end{array}$ & $\begin{array}{c}K \\
{[\mathrm{MeV}]}\end{array}$ & $\begin{array}{c}S \\
{[\mathrm{MeV}]}\end{array}$ & $\begin{array}{c}L \\
{[\mathrm{MeV}]}\end{array}$ & $\begin{array}{l}R_{1.4} \\
{[\mathrm{~km}]}\end{array}$ & $\begin{array}{l}M_{\max } \\
{\left[M_{\odot}\right]}\end{array}$ & $\overline{\text { Ref }}$ \\
\hline DD2 & $\mathrm{NSE}+\mathrm{RMF}$ & 0.1491 & 16.02 & 243 & 31.67 & 55.04 & 13.2 & 2.42 & 53,54 \\
\hline LS220 & SNA + Liquid droplet Skyrme & 0.1550 & 16.00 & 220 & 28.61 & 73.82 & 12.7 & 2.06 & 9 \\
\hline SFHo & $\mathrm{NSE}+\mathrm{RMF}$ & 0.1583 & 16.19 & 245 & 31.57 & 47.10 & 11.9 & 2.06 & 55 \\
\hline & experiments and observations & $0.15-0.16$ & $\sim 16$ & $220-315$ & $28.5-34.9$ & $30.6-86.8$ & $11.2-13.4$ & $>1.97$ & \\
\hline
\end{tabular}

Table 2. Fiducial binary neutron star configurations. We report EOS, individual gravitational masses and maximum rest-mass density of the initial data in units of $\rho_{0} \simeq 2.7 \times$ $10^{14} \mathrm{~g} \mathrm{~cm}^{-3}$. All simulations were performed with a maximal grid resolution of $185 \mathrm{~m}$, using the MO neutrino-transport approximation. Two simulations include the effect of physical viscosity and are indicated with "V" (see text for more details.)

\begin{tabular}{llcc}
\hline \hline Simulation & EOS & $\left(M_{1}, M_{2}\right)$ & $\left(\rho_{\mathrm{NS} 1}, \rho_{\mathrm{NS} 2}\right)$ \\
\hline DD2_M136136 & DD2 & $(1.36,1.36)$ & $(2.1,2.1)$ \\
LS220_M135135 & LS220 & $(1.35,1.35)$ & $(2.6,2.1)$ \\
LS220_M135135V & LS220 & $(1.35,1.35)$ & $(2.6,2.6)$ \\
LS220_M140120V & LS220 & $(1.40,1.20)$ & $(2.7,2.3)$ \\
SFHo_M135135 & SFHo & $(1.35,1.35)$ & $(3.0,3.0)$ \\
\hline \hline
\end{tabular}

pseudo-spectral code [56] and specify the initial separation between the NS to at least $40 \mathrm{~km}$, corresponding to $\sim 2-3$ orbits before merger. The EOS used for the initial data are constructed from the minimum temperature slice $(T \sim 0.5-0.1 \mathrm{MeV})$ of the EOS table used for the evolution assuming neutrino-less beta-equilibrium. To create the initial data tables we also subtract from the pressure the contribution of photon radiation, which dominates at the lowest densities due to the assumption of constant temperature. Initial data properties are summarized in Tab. 2.

The initial data are evolved according to Einstein's general relativistic equations for the spacetime in the $(3+1)$ form described in [57,58] and coupled to general relativistic hydrodynamics. The $(3+1) \mathrm{D}$ simulations span the merger and the remnant evolution for a timescale of at least 20-30 ms. We use the WhiskyTHC code [59, 36, 60, 61]; all the technical details are given in [23. All simulations involving the LS220 EOS and simulation DD2_M136136 are reported here for the first time. All our simulations domain covers a cube of $3,024 \mathrm{~km}$ in diameter whose center is at the center of mass of the binary. Our code uses BergerOliger conservative adaptive mesh refinement (AMR; 62 ) with sub-cycling in time and refluxing [63,64 as provided by the Carpet module of the Einstein Toolkit [65]. We setup an AMR grid structure with 7 refinement levels. The finest refinement level covers both NSs during the inspiral and the remnant after the merger, and has a typical resolution of $h \simeq 185 \mathrm{~m}$.
For a subset of binaries, we use the general-relativistic large eddy simulations method (GRLES; 66]) to explore the impact of subgrid-scale turbulent angular momentum transport. The turbulent viscosity is parametrized as $\sigma_{T}=$ $\ell_{\text {mix }} c_{s}$, where $c_{s}$ is the sound speed, and $\ell_{\text {mix }}$ is a free parameter that sets the intensity of the turbulence. In the context of accretion disk theory turbulent viscosity is usually expressed in terms of a dimensionless constant $\alpha$ related to $\ell_{\text {mix }}$ through the relation $\ell_{\text {mix }}=\alpha c_{s} \Omega^{-1}$, where $\Omega$ is the angular velocity of the fluid [67]. Recently, very high resolution general relativistic magnetohydrodynamics simulations of a NS merger were reported by 68, who used sufficiently high seed magnetic fields $\left(10^{15} \mathrm{G}\right)$ to resolve the MRI in the merger remnant. Averaged $\alpha$ values for different rest-mass density shells were also provided. In our models, we combine values of $c_{s}$ and $\Omega$ directly obtained during the simulations with their estimate of $\alpha$ to compute $\ell_{\text {mix }}$ as a function of density.

The high temperatures reached during the merger lead to the copious production of neutrinos of all flavors. Electron (anti)neutrino production rates due to electron and positron captures on free protons and neutrons, respectively, are characterized by a $T^{5}$ dependence on matter temperature (e.g., 69]). Thermal processes producing all kinds of neutrinos have an even stronger dependence, $\sim T^{7}$ (e.g., [70]). For densities in excess of $\rho_{\text {lim }} \sim 10^{12} \mathrm{~g} \mathrm{~cm}^{-3}$ and temperatures above a few $\mathrm{MeV}$, the neutrino mean free path becomes smaller than the typical length scales of the system, and the diffusion timescale significantly exceeds the dynamical timescales. Under these conditions, Deutrino trapping occurs and neutrinos form a gas thermally and dynamically coupled with the baryonic fluid. Diffusing neutrinos are eventually emitted at the last scattering surface (also called neutrino surface), located at a density close to $\rho_{\mathrm{lim}}$, and propagate further in optically thin conditions.

The compositional and energy changes in the matter due to weak reactions during the simulations are treated using a leakage scheme that tracks reactions involving electron $\nu_{e}$ and anti-electron type $\bar{\nu}_{e}$ neutrinos separately 71 , 72. Heavy-lepton neutrinos are treated as a single effective species labeled as $\nu_{x}$. The reactions included in the simulations are listed in Tab. 3 the precise formulas implemented for neutrino production and absorption rates and for scattering opacities can be found in the references listed there. The total neutrino opacities are used to compute an estimate to the optical depth following the scheme of 73 . 
Table 3. Weak reaction rates and references for their implementation. We use the following notation: $\nu \in\left\{\nu_{e}, \bar{\nu}_{e}, \nu_{x}\right\}$ denotes a neutrino species, $\nu_{x}$ any heavy-lepton neutrinos, $N \in\{n, p\}$ denotes a nucleon, and $A$ denotes a nucleus.

\begin{tabular}{lr}
\hline \hline Reaction & Ref. \\
\hline$\nu_{e}+n \leftrightarrow p+e^{-}$ & 78 \\
$\bar{\nu}_{e}+p \leftrightarrow n+e^{+}$ & 78 \\
$e^{+}+e^{-} \rightarrow \nu+\bar{\nu}$ & 74 \\
$\gamma+\gamma \rightarrow \nu+\bar{\nu}$ & 74 \\
$N+N \rightarrow \nu+\bar{\nu}+N+N$ & 779 \\
$\nu+N \rightarrow \nu+N$ & 74 \\
$\nu+A \rightarrow \nu+A$ & {$[80$} \\
\hline \hline
\end{tabular}

The optical depth and local opacity are then employed to calculate the neutrino diffusion timescale, as described in 74, 4. The neutrino emission rate from optically thick regions is then computed as the ratio between the local neutrino densities and the diffusion timescale. For the former quantity, we assume that the neutrinos follow FermiDirac distributions with chemical potentials obtained assuming beta-equilibrium with thermalized neutrinos as in [69]. Free-streaming neutrinos are emitted at an average energy and then evolved according to the M0 scheme introduced in 72,4. The M0 scheme is less sophisticated that the frequency-integrated M1 scheme adopted by others 75, 76. However, it is has the advantage of computational efficiency, it incorporates an approximate treatment of gravitational and Doppler effects, and is well adapted to the geometry found in NS mergers. In particular, it is not affected by the well-known radiation shock artifact that plagues the M1 scheme [77.

\subsection{Modelling of trapped neutrinos}

Despite the fact that trapped neutrino density is employed to compute emission rates from optically thick regions, the neutrino treatment adopted in our simulations does not explicitly model the presence of a trapped component. The temperature- and composition-dependent weak equilibrium that establishes between the baryonic and leptonic fluids for $\rho \gtrsim \rho_{\text {lim }}$ could potentially change the matter properties at those densities. For example, we presently neglects the pressure and internal energy contributions, as well as possible changes in the electron fraction, due to the presence of these neutrino gases.

In order to explore this effect and to assess its relevance, we postprocess the results of our simulations by computing the thermodynamical conditions of the fluid in presence of a neutrino gas in weak and thermal equilibrium. For consistency with the underlying simulations, we consider (anti)neutrino of all flavors modelled as three independent species: $\nu_{e}, \bar{\nu}_{e}$, and $\nu_{x}$. Since neutral current reactions involving $\nu_{x}-\bar{\nu}_{x}$ pairs decouple at significantly larger densities than charged current reactions involving $\nu_{e}$ and $\bar{\nu}_{e}$, we distinguish between $\rho_{\text {lim,e }}=5 \times 10^{11} \mathrm{~g} \mathrm{~cm}^{-3}$ for both $\nu_{e}$ and $\bar{\nu}_{e}$, and $\rho_{\mathrm{lim}, \mathrm{x}}=5 \times 10^{12} \mathrm{~g} \mathrm{~cm}^{-3}$ for $\nu_{x}$.
2 We first promote the electron fraction and the internal energy density obtained by the simulations and measured in the local comoving frame to the lepton and total (i.e., fluid+neutrinos) internal energy density:

$$
\begin{aligned}
Y_{\mathrm{e}, \operatorname{sim}} & \rightarrow Y_{l} \\
e_{\mathrm{sim}} & \rightarrow u .
\end{aligned}
$$

Indeed, the leakage scheme prescriptions evolve the lepton fraction and total internal energy, rather than the electron fraction and the fluid internal energy (see, e.g., 81). For a given matter density $\rho$, the electron fraction and the fluid energy obtained in the simulations univocally identify a temperature through the EOS, $T_{\text {sim. }}$. We then compute the electron fraction and the temperature at equilibrium, $\left(Y_{e, \text { eq }}, T_{\text {eq }}\right)$, solving the following set of equations:

$$
\begin{gathered}
Y_{l}=Y_{e, \text { eq }}+Y_{\nu_{e}}\left(Y_{e, \text { eq }}, T_{\text {eq }}\right)-Y_{\bar{\nu}_{e}}\left(Y_{\mathrm{e}, \text { eq }}, T_{\text {eq }}\right) \\
u=e\left(Y_{e, \text { eq }}, T_{\text {eq }}\right)+\frac{\rho}{m_{\mathrm{b}}}\left[Z_{\nu_{e}}\left(Y_{e, \text { eq }}, T_{\text {eq }}\right)+\right. \\
\left.+Z_{\bar{\nu}_{e}}\left(Y_{e, \text { eq }}, T_{\text {eq }}\right)+4 Z_{\nu_{x}}\left(T_{\text {eq }}\right)\right] \\
0=\quad \eta_{\nu_{e}}\left(Y_{e, \text { eq }}, T_{\text {eq }}\right)-\eta_{e}\left(Y_{e, \text { eq }}, T_{\text {eq }}\right)+ \\
-\eta_{\mathrm{p}}\left(Y_{e, \text { eq }}, T_{\text {eq }}\right)+\eta_{\mathrm{n}}\left(Y_{e, \text { eq }}, T_{\text {eq }}\right) .
\end{gathered}
$$

In the previous equations, $Y_{\nu_{i}}$ and $Z_{\nu_{i}}$ represent the neutrino particle and energy fractions, respectively, while $\eta_{i}=\mu_{i} / T$ is the degeneracy parameter for the $i$ species. They are computed assuming weak and thermal equilibrium between all the modelled species. The former is ensured by the equilibrium relationship between the relativistic chemical potentials $\mu$, i.e. $\mu_{\nu_{e}}=\mu_{e}+\mu_{p}-\mu_{n}$. The latter is given by the assumption that the neutrino and matter temperature coincide. In particular, we compute

$$
\begin{aligned}
& Y_{\nu_{i}}\left(\rho, Y_{e}, T\right)=\frac{4 \pi m_{\mathrm{b}}}{\rho(h c)^{3}}\left(k_{\mathrm{B}} T\right)^{3} F_{2}\left(\eta_{\nu_{i}}\right) \exp \left(-\frac{\rho_{\mathrm{lim}, \mathrm{i}}}{\rho}\right)(4) \\
& Z_{\nu_{i}}\left(\rho, Y_{e}, T\right)=\frac{4 \pi m_{\mathrm{b}}}{\rho(h c)^{3}}\left(k_{\mathrm{B}} T\right)^{4} F_{3}\left(\eta_{\nu_{i}}\right) \exp \left(-\frac{\rho_{\mathrm{lim}, \mathrm{i}}}{\rho}\right)(5)
\end{aligned}
$$

where $F_{k}(x)$ is the Fermi function of order $k$, and we further assume that $\mu_{\bar{\nu}_{e}}=-\mu_{\nu_{e}}$ and $\mu_{\nu_{x}}=0$. The density dependent exponential term ensures that trapped neutrinos are only present for $\rho \gtrsim \rho_{\text {lim }}$ (see e.g. 82 ). Trapped neutrinos do provide a pressure as an ultra-relativistic gas, i.e. $P_{\nu}=\left(\rho Z_{\nu}\right) /\left(3 m_{\mathrm{b}}\right)$. We thus compare the pressure obtained in the simulation,

$$
P_{\text {sim }} \equiv P_{\text {fluid }}\left(\rho, Y_{e, \operatorname{sim}}, T_{\text {sim }}\right),
$$

with the total pressure at equilibrium,

$$
\begin{aligned}
P_{\text {eq }} \equiv & P_{\text {fluid }}\left(\rho, Y_{e, \text { eq }}, T_{\text {eq }}\right)+ \\
& +\frac{\rho}{3}\left(Z_{\nu_{e}}+Z_{\bar{\nu}_{e}}+4 Z_{\nu_{x}}\right)\left(Y_{e, \text { eq }}, T_{\text {eq }}\right) .
\end{aligned}
$$

\section{Thermodynamical evolution}

We now discuss results for the four fiducial binary neutron star mergers reported in Tab. 2, The binaries have

\footnotetext{
2 Note that we do not include the presence of a scattering atmosphere, which could be relevant for $\nu_{x}$ neutrinos.
} 


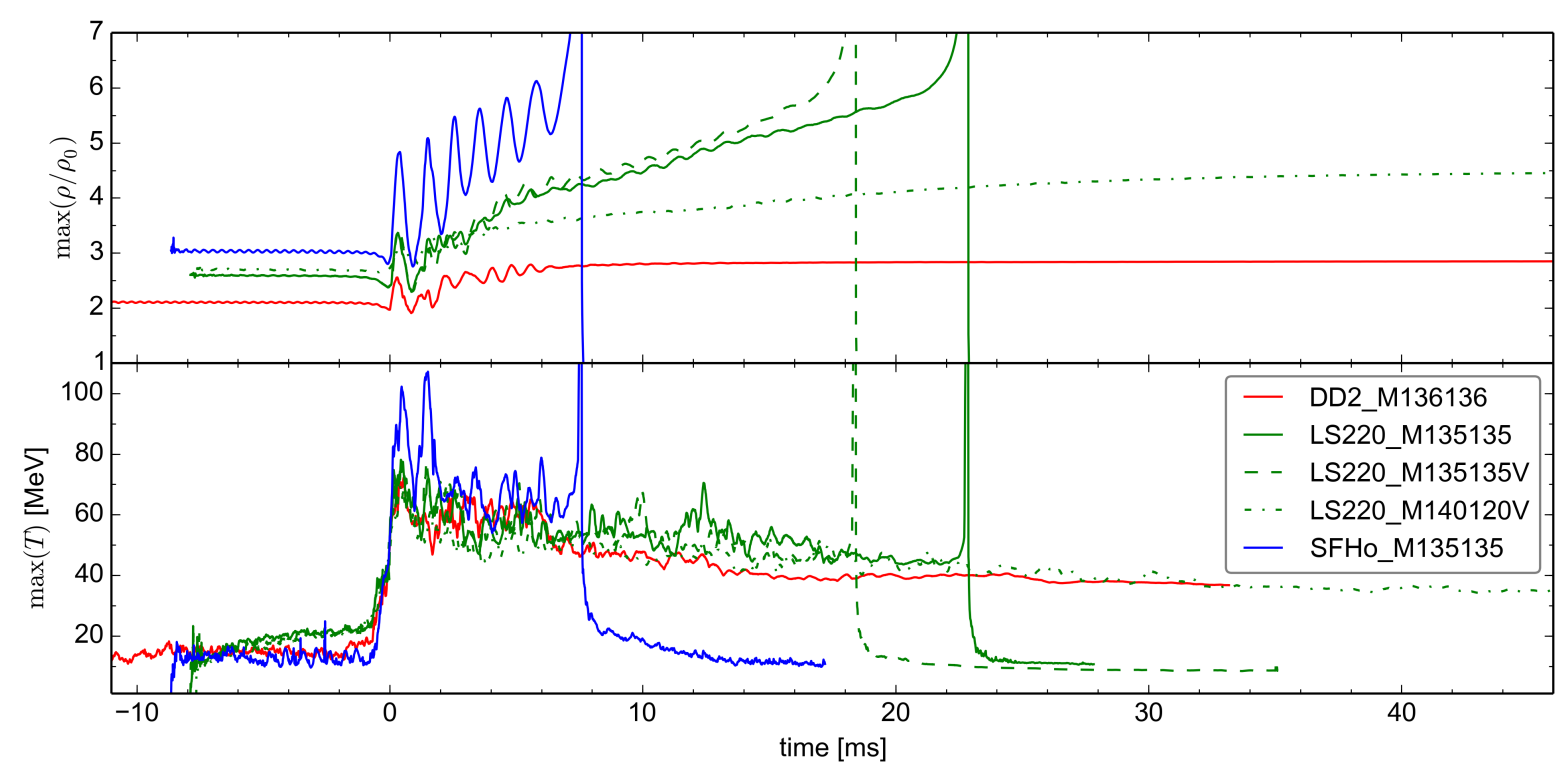

Fig. 1. Evolution of the maximum rest-mass density (top) and of the maximum temperature (bottom) for all the binaries considered in this work. Peak temperatures correspond the first bounce of the NS cores, and are reached at typical densities $\rho \sim \rho_{0}$. Collapse to black hole happens for LS220_M135135 and SFHo_M135135; during collapse the maximum density reaches $(\max \rho) \gtrsim 5 \rho_{0}$.

masses and EOS compatible with GW170817 31 and, correspondingly, the maximal densities in each NS are $2-3$ times the nuclear saturation point, $\rho_{\mathrm{NS}_{1,2}} \sim 2-3 \rho_{0}$, with softer EOS having larger maximum densities.

The maximum density and temperature evolutions for each model are reported in Fig. 1. The moment $t=0$ corresponds to the peak of the gravitational-wave amplitude (i.e. the end of the chirp signal) and it is conventionally refereed as moment of merger (or shortly merger, where it cannot be confused with the merger-phase of the coalescence) [17. During the last orbits $(t<0)$ the maximum density and temperature are approximately constant, the latter being $T \sim 10 \mathrm{MeV}$. These high temperature are reached close to the surface of the stars and are a numerical artifact.

At $t \sim 0$ the two NS cores merge, the maximum density increases rapidly up to $\sim 1.5 \rho_{\mathrm{NS}}$ and temperature increases due to (physical) hydrodynamical shocks and matter compression. In the considered binaries, no prompt collapse happens and the two NS cores bounce multiple times on a timescale of few milliseconds. During the first of these bounces, the temperature peaks at $70-100 \mathrm{MeV}$ and most of the dynamical ejecta is expelled [83,84,4. The core bounces are more violent in binaries with softer EOS, and produce larger density and temperature oscillations. Note that binary remnants are closer to the gravitational collapse instability point for softer EOSs 1, 85, 86. Hot material from the collisional interface between the NSs is squeezed out of the remnant and settles into an accretion disk [4. The remnant is still deformed into a bar $(m=2$ mode) and spiral arms are launched inside the disk. On a timescale of $10-20 \mathrm{~ms}$, the characteristic timescale of the GW transient, binaries with softer EOS collapse to a rotating black hole with typical dimensionless Kerr parameter of $\sim 0.7-0.8$ 3] 19. Binaries with stiffer EOS generate long-lived NS remnants whose dynamics is determined on timescales much longer than those presently simulated in numerical relativity [23].

Let us discuss the thermodynamical conditions experienced by the matter during the different phases of the merger. For the moment, we do not include the effect of trapped neutrinos at $\rho>\rho_{\text {lim }}$, but we simply consider the outcome of our simulations. Under the assumption that matter is always in Nuclear Statistical Equilibrium (NSE), we focus on matter density, $\rho$, temperature, $T$, and electron fraction, $Y_{e}$. We consider 3D spatial hypersurfaces at specific coordinate times and produce histograms of the baryonic-mass as distributed at different $\rho, T$, and $Y_{e}$, i.e. $M_{\mathrm{b}}\left(\rho, T, Y_{e}\right)$. We used the following ranges:

$$
\begin{aligned}
\rho & \in\left[10^{4}, 10^{16}\right] \mathrm{g} \mathrm{cm}^{-3} \\
T & \in\left[10^{-2}, 10^{2}\right] \mathrm{MeV} \\
Y_{e} & \in[0,0.5],
\end{aligned}
$$

binning them in $120 \times 50 \times 50$ intervals. For the density and temperature, the bins are uniformly displaced in the logarithm, while we use a linear scale for the electron fraction. The artificial atmosphere of the simulations is set to density level $\rho_{\text {atm }} \sim 10^{4} \mathrm{~g} \mathrm{~cm}^{-3}$. Similar plots have been presented in [87,88] for core-collapse supernova simulations, in [29, 89] for BNS mergers, and in 90 for BNS merger aftermath ${ }^{3}$. However, a detailed discussion is, to the best of our knowledge, missing in the literature.

\footnotetext{
3 Note the LS220 simulations presented here are a longer and higher resolution versions of those shown in 89 .
} 
The five rows of Figs. $\left.2 \sqrt{6}\right|^{4}$ refer to snapshots covering the entire available binary evolution. Moving from top to bottom: (1) early time during the orbital phase, (2) the time corresponding to the temperature peak, (3) about 3-4 milliseconds after the temperature peak, (4) a later time close to collapse (if collapse happens), (5) time close to simulation end. Each row shows a snapshot of restmass density on the orbital plane and two thermodynamical condition histograms in which two of three variables $\left((\rho, T)\right.$ and $\left(\rho, Y_{e}\right)$, respectively) are shown and the third is integrated over its range.

During the last orbits (snapshots (1)) most of the baryon mass is clearly distributed around the maximum NS densities at $T \sim 1-3 \mathrm{MeV}$, with an electron fraction still close to the cold, $\nu$-less weak equilibrium, $0.01 \lesssim Y_{e} \lesssim 0.12$. In all models we recognize the typical increase of $\tilde{Y}_{e}$ moving from $\rho \sim \rho_{0}$ to a few times $\rho_{0}$ due to the increase in the symmetry energy. The highest temperatures are reached at densities $\rho \sim 10^{12} \mathrm{~g} \mathrm{~cm}^{-3}$, but as mentioned above this is a numerical artifact.

After merger $(t \gtrsim 0$, snaphshots (2)), the innermost part of the merging cores, $\rho \gtrsim \rho_{\mathrm{NS}}$, does not experience violent shocks $\left(s \lesssim 1 k_{\mathrm{B}}\right.$ baryon $^{-1}$, where $s$ is the specific matter entropy). Thus the increase of the maximum density in the center of the remnant is associated with a marginal increase in the temperature (up to a few tens of $\mathrm{MeV}$ ), mainy due to matter compression of degenerate nuclear matter well above nuclear saturation density. Indeed, most of the matter in the cores resides below the semi-degenerate transition line represented by

$$
T=T_{\mathrm{F}}(\rho)=\frac{\hbar^{2}}{2 m_{b}}\left(\frac{3 \pi^{2} \rho}{m_{b}}\right)^{2 / 3}
$$

where $T_{\mathrm{F}}$ is the Fermi temperature of an ideal baryon gas of mass $m_{b}$. At merger $(t \sim 0)$ the electron fraction at the highest densities is still frozen to the initial $\nu$-less, cold weak equilibrium value. Due to the high opacities of these regions during and after the merger, $Y_{e}$ does not change significantly on the simulated timescale due to the emission of neutrinos. Only later, when the cores experience a significant compression and mixing, the $Y_{e}$ profiles become more uniform for $\rho \gtrsim \rho_{\mathrm{NS}}$.

On the contrary, the densities at which $T$ peaks correspond to the regions close to the binary center of mass where shocks are generated and material is pushed outwards (snaphshots (2)-(3)). The highest temperatures are reached at densities slightly below $\rho_{\mathrm{NS}}$ and characterized by the lowest $Y_{e}$. In particular, the DD2 model peaks at $\sim 70 \mathrm{MeV}, \mathrm{LS} 220$ at $\sim 80 \mathrm{MeV}$, and SFHo at $\sim 100 \mathrm{MeV}$. This matter, initially located in the outer layers of the two cores, expands into the spiral arms. The temperature lowers as the spiral-arms expand and decompress, forming an envelope around the merging cores. As a consequence of the fast expansion, the temperature drops to $\sim 10 \mathrm{MeV}$ as the density reaches a few times $10^{13} \mathrm{~g} \mathrm{~cm}^{-3}$ (snapshot

4 Time-dependent (animated) versions can be found at http://www. computational-relativity.org/
(3)). The EOS is dominated by the non-degenerate nucleons $\left(T \gtrsim T_{\mathrm{F}}\right)$ and the matter expanding adiabatically satisfies the relation $T^{3} / \rho^{2} \approx$ const 5

In terms of the spatial distribution of $\rho$ and $T$, the merging cores have a peculiar structure with two rotating hot spots, displaced by an angle of $\sim \pi / 2$ with respect to the densest regions corresponding to the NS cores (e.g. 91]). The expansion of the spiral arms, in combination with their fast orbital motion, produces a disk structure around the forming massive NS. The fast motion of the merging cores and of spiral arms inside this newly formed envelope heats it up, mainly via shocks. The subsequent increase of entropy determines a spread in the $\rho-T$ diagram. The EOS is dominated by the nonrelativistic baryons, $T \propto\left(\exp (2 s) / \rho^{2}\right)^{1 / 3}$ (see, e.g., 92]) and matter experiences a temperature increase up to a few tens of MeV (snapshots (3) and (4)). This behaviour explains the peak structure and evolution observed in the $\rho-T$ diagram, where a maximum temperature of a several tens of $\mathrm{MeV}$ is reached by a substantial amount of matter with density around or immediately below nuclear saturation density. The corresponding electron fraction is significantly larger $(\sim 0.05-0.10)$ than the low $Y_{e}$ expected from $\nu$-less, cold weak equilibrium $(\lesssim 0.05)$. This is only partially due to an excess of electron antineutrinos emission (see below), while it traces back the origin of this matter to larger densities where the increase of the symmetry energy produces larger $Y_{e}$ already in $\nu$-less weak equilibrium conditions.

Shocks inside the disk produce further matter expansion (snaphshots (3)-(5)). As soon as the density drops below $10^{10} \mathrm{~g} \mathrm{~cm}^{-3}$ and the temperature becomes of the order of a few $\mathrm{MeV}$, the EOS is dominated by the relativistic electrons and photons. Thus, adiabatic expansion follows the relation $T^{3} / \rho \approx$ const $\propto s$, typical of ultrarelativistic gases.

If the forming massive NS does not collapse (as in the DD2 case, Fig. 22), the cores completely merge on a timescale of several ms and the hot spots become a spherical annulus at an intermediate density $\sim \rho_{0}$ between the denser core and the more diluite envelope, see Fig. 8. Since this time interval corresponds to several orbital timescales, the envelope around the remnant has reached a relatively homogeneous configuration and the spread in the $\rho-T$ plane has significantly reduced. Moreover, an inspection of the $\rho-Y_{e}$ planes reveals the effect of weak reactions on the disk composition: at the location where the last scattering neutrino surface is expected to be $\left(\rho \sim \rho_{\text {lim }}\right)$, the captures of positrons and streaming $\nu_{e}$ on free neutrons significantly increases the electron fraction.

On the other hand, if a black hole forms (as in the LS220 and SFHo cases, see the fifth row of Figs. 3 36, the evolution of the thermodynamical conditions inside the

${ }^{5}$ Thermodynamics' first principle for an adiabatic trasformation of an ideal gas in a volume $V$ is $d U / V=+p d \rho / \rho$ with $U$ the internal energy and $d U \propto d T$. For a non-relativistic gas $P=2 / 3 U / V$ and for a relativisitc gas $P=1 / 3 U / V$. Hence, in the former case $d \ln T=2 / 3 d \ln \rho$, and in the latter $d \ln T=1 / 3 d \ln \rho$. 
remnant changes substantially. Within a ms, the formation of an apparent horizon removes all the high density part of the system, down to densities of $10^{12} \mathrm{~g} \mathrm{~cm}^{-3}$, leaving a rather cold disk $(T \lesssim 10 \mathrm{MeV})$ with a significantly reprocessed electron fraction, i.e. $Y_{e} \sim 0.25$.

In Fig. 7. we present histograms of the thermodynamical conditions from the third and fifth snaphshots for all the models presented in Figs. 2, 6, binned in density and specific entropy, $s$. For the latter, we consider 50 logarithmically spaced bins in the interval

$$
s \in[0.1,100] k_{\mathrm{B}} \text { baryon }^{-1} .
$$

For all models, the cold unshocked cores are characterized by the presence of low entropy material $\left(s \lesssim 2 k_{\mathrm{B}}\right.$ baryon $\left.^{-1}\right)$ for matter densities in excess of a few times $\rho_{0}$. Matter squeezed by the merging cores and expanding inside the forming disk is subjects to intense hydrodynamical shocks that increase matter entropy up to a few $k_{\mathrm{B}}$ baryon $^{-1}$. The combined effect of matter expansion and shocks resulting from the repeated core bounces produces a transient phase where the envelope engulfing the cores has a large spread in entropy (see, for example, the left panel of the DD2_M136136 cases). However, on a timescale of a few dynamical periods, the action of the spiral arms on the innermost part of the disk increases the entropy of the colder streams, producing a tighter correlation between the matter density and entropy. The bulk of the remnant outside the merging cores has $2<s\left[k_{\mathrm{B}}\right.$ baryon $\left.^{-1}\right] \lesssim 10$ for matter density decreasing from $10^{14}$ down to $10^{10} \mathrm{~g} \mathrm{~cm}^{-3}$. Looking at the matter distribution inside this density interval, we conclude that softer EOSs present a remnant characterized by larger values of the entropy (i.e. $\Delta s \sim$ $2 k_{\mathrm{B}}$ baryon $\left.^{-1}\right)$. This is a consequence of the stronger shocks that characterize more violent mergers from more compact NSs. If the EOS is stiffer and the massive NS survives long enough, shock expansion inside the low density part of the disk increases its entropy, up to $20 k_{\mathrm{B}}$ baryon $^{-1}$. On the other hand, if a black hole forms, the lack of a persistent source of shocks partially prevents the disk from reaching a tight $\rho-s$ correlation (as visible in the LS220 and SFHo equal mass cases).

Apart from the differences due to the different fates of the remnant, all five fiducial models show a similar qualitative behavior, and the major features described above do not depend on the specific EOS nor on the binary mass ratio. Nevertheless, quantitative differences and temporary features can be noticed depending on the specific model. Temperature and entropy are, on average, larger for models employing a softer EOS. This is a results of the more violent collision that characterize more compact NSs. Models employing turbulent viscosity show immediately after merger a larger spread in the density-temperature histogram, as well as in the density-entropy one. However, they require less time to produce disks with homogeneous properties. In the asymmetric model LS220_M140120, the cores merge in a very asymmetric way. In particular, the core of the lighter NS is tidally deformed by the core of the heavier one and its matter is subject to a violent temperature increase during its decompression (see the second raw of Fig. 5). However, on a longer timescale, the outer part of the lighter core contributes to the formation of the hot envelope around the merging cores and the evolution of all termodynamical quantities resembles the one of more symmetric mergers.

Finally, it is interesting to note that our histograms track the evolution of the dynamical ejecta in its different components. Immediately after merger, a few ejection episodes develops from matter with initially low temperature (a few $\mathrm{MeV}$ ), low $Y_{e}(\lesssim 0.1)$, and low entropy $\left(\lesssim 10 k_{B}\right)$. They expands very fast according to $T^{3} / \rho^{2} \sim$ const, down to densities at the edge of our domain. Positron and electron neutrino captures on free $n$ increase $Y_{e}$ during the expansion, but only marginally $\left(Y_{e} \lesssim 0.25\right)$. We tentatively identify this ejecta as the tidal component. After $\sim 1$ millisecond, ejection episodes are also visible from the high-temperature part of our histograms (corresponding to $s \gtrsim 10 \mathrm{k}_{\mathrm{B}}$ baryon $^{-1}$ ), expanding at the same rate. In the $T-Y_{e}$ plane, this ejecta presents a much broader distribution in $Y_{e}$, up to $Y_{e} \lesssim 0.4$. We identify this ejecta as the shock-driven one, whose polar component is more significantly influenced by neutrino absorption.

\section{Influence of neutrino trapping on matter}

We now move to the discussion of the potential effect of trapped neutrinos using the approached described in Section 2.1. We first re-write the expression of the neutrino fractions, Eqs. 4.5, in terms of the Fermi temperature, Eq. 8, neglecting the exponential cut in density:

$$
\begin{aligned}
& Y_{\nu_{i}}\left(\rho, Y_{e}, T\right)=\frac{3 \sqrt{2}}{8}\left(\frac{T}{T_{\mathrm{F}}}\right)^{3 / 2}\left(\frac{k_{\mathrm{B}} T}{m_{b} c^{2}}\right)^{3 / 2} F_{2}\left(\eta_{\nu_{i}}\right) \\
& Z_{\nu_{i}}\left(\rho, Y_{e}, T\right)=\left(k_{\mathrm{B}} T \frac{F_{3}\left(\eta_{\nu_{i}}\right)}{F_{2}\left(\eta_{\nu_{i}}\right)}\right) Y_{\nu_{i}}\left(\rho, Y_{e}, T\right)
\end{aligned}
$$

Since baryons are always non relativistic, $k_{B} T \ll m_{b} c^{2}$, significant neutrino fractions require high temepratures for matter in non-degenerate conditions $\left(T \gg T_{\mathrm{F}}\right)$ and/or highly degenerate neutrino conditions $\left(\eta_{\nu} \gg 1\right)$.

We have repeated the analysis of the histrograms of the thermodynamical conditions presented in Section 3 including the effects of trapped neutrinos for $\rho>\rho_{\mathrm{lim}}$. Since the variations in $Y_{e}$ and $T$ are only minors (see below) and the histograms present already a significant spread in all variables, we conclude that the inclusion of trapped neutrinos does not qualitatively changes our previous analysis.

In Fig. 9 we present the results of our post-processing analysis for a snapshot of the DD2_M136136 simulation taken $\sim 11 \mathrm{~ms}$ after merger (i.e. close to the peak temperature) along the equatorial plane. In the top panels, we show the rest-mass density (left), matter temperature (middle) and electron fraction (right) as obtained by the simulation. In the bottom panels, we present $Y_{\nu_{e}}$ (left), $Y_{\bar{\nu}_{e}}$ (middle), and $Y_{\mathrm{e}, \text { sim }}-Y_{\mathrm{e}, \mathrm{eq}}=Y_{\nu_{e}}-Y_{\bar{\nu}_{e}}$ (right) as obtained in our post-processing analysis.

Deep inside the remnant, where densities are in excess of a few times nuclear saturation density, $\rho \gtrsim 3 \rho_{0}$, 
the temperature is a few $\mathrm{MeV}$ high. Under such conditions, $T \ll T_{\mathrm{F}}$ (i.e. $\mu_{i} \gg T$ for neutrons, protons, and electrons, and thermal effects are negligible). Since matter conditions are still close to the initial cold, $\nu$-less, weak equilibrium, we find $\left|\eta_{\nu_{i}}\right| \lesssim 3$, and $F_{2}\left(\eta_{\nu_{i}}\right) \lesssim 20$ (see e.g. [93], appendix). Thus, the production of electron flavor (anti)neutrinos is suppressed and $Y_{\mathrm{e}, \mathrm{eq}} \approx Y_{\mathrm{e}, \mathrm{sim}}$. Since $Y_{e} \lesssim 0.1$, neutrons are more degenerate than protons and any increase in temperature decreases more significantly $\mu_{p}$ than $\mu_{n}$. Thus, $\eta_{\nu_{e}} \lesssim 0$. This effect is partially compensated by the increase of $\mu_{e}$ due to matter compression. Overall, we find that $-3 \lesssim \eta_{\nu_{e}} \lesssim-1,10^{-6} \lesssim Y_{\nu_{e}} \lesssim 10^{-4}$, $10^{-4} \lesssim Y_{\bar{\nu}_{e}} \lesssim 10^{-3}$, and $10^{-6} \lesssim Y_{\nu_{x}} \lesssim 10^{-4}$, such that $Y_{\nu_{e}}<\widetilde{\widetilde{Y}}_{\nu_{x}}<{\widetilde{Y_{\bar{\nu}}}}_{\bar{\nu}_{e}}$ locally.

At lower densities, $10^{14} \mathrm{~g} \mathrm{~cm}^{-3} \lesssim \rho \lesssim 4 \times 10^{14} \mathrm{~g} \mathrm{~cm}^{-3}$, the temperature increases up to a few tens of $\mathrm{MeV}$, due to the presence of the hot annulus outside the merging cores. While the electrons are still highly degenerate, the neutrons and (more significantly) the less abundant protons becomes mildy degenerate $\left(T \gtrsim T_{\mathrm{F}}\right)$. The (negative) chemical potential of electron neutrinos decreases, but not the neutrino degeneracy parameter, due to the higher temperatures: in this region we find that $-2 \lesssim \eta_{\nu_{e}} \lesssim-1$. Under these conditions, a significant electron antineutrino gas forms $Y_{\bar{\nu}_{e}} \sim 0.02$, followed by $Y_{\nu_{x}} \lesssim 0.01$, while electron neutrinos are still suppressed by degeneracy $\left(Y_{\nu_{e}} \lesssim 10^{-3}\right)$. Due to the dominant presence of $\bar{\nu}_{e}$, the initial $Y_{\mathrm{e}, \mathrm{sim}}$ $(\sim 0.06)$ increases by $Y_{\bar{\nu}_{e}}$, i.e. $Y_{\mathrm{e}, \mathrm{eq}} \approx Y_{\mathrm{e}, \mathrm{sim}}+Y_{\bar{\nu}_{e}}$, to guarantee lepton number conservation.

At densities below $10^{14} \mathrm{~g} \mathrm{~cm}^{-3}$ the decrease in temperature related with matter expansion produces less significant deviations from the initial cold $\nu$-less weak equilibrium and all neutrino fractions become negligible inside the cold $(T \lesssim 10 \mathrm{MeV})$, unshocked streams. In particular, $\eta_{\nu_{e}} \gtrsim 1$ and $\nu_{e}$ dominate over $\bar{\nu}_{e}$. However, due to the low temperatures, $T<T_{\mathrm{F}}$ and $Y_{\nu_{e}} \sim 10^{-3}$. At the same time, spiral arms moving inside this cold and less dense matter produce shockes that heat-up matter at the arm interfaces. Inside these regions, temperatures in excess of $10 \mathrm{MeV}$ for matter that decompresses down to a few times $10^{12} \mathrm{~g} \mathrm{~cm}^{-3}$ (thus, $T \sim$ several $T_{\mathrm{F}}$ ) lead to $\eta_{\nu_{e}} \sim-1$, producing trapped neutrino gases with $Y_{\bar{\nu}_{e}} \lesssim 0.03, Y_{\nu_{x}} \lesssim$ 0.01 , and $Y_{\nu_{e}} \sim$ a few $10^{-3}$. As a results, $Y_{e, \text { eq }}$ increases with respect to $Y_{e, \mathrm{sim}}$.

The appearance of a trapped neutrino gas is done at the expences of the fluid internal energy, formed by relativistic electrons, positrons, photons, and non-relativistic baryons. Since thermodynamical stability requires $\partial e / \partial T>$ $0, T_{\text {eq }}<T_{\text {sim }}$. In the top panel of Fig. 10 we present the ratio between the fluid pressure after and before the postprocessing for the same configuration presented in Fig. 9 The inclusion of trapped neutrinos reduces the temperature down to $\sim 93 \%$ of the simulation value in the hottest regions of the systems. Neutrinos are intrinsecally ultrarelativistic and for them $P_{\nu} \propto Z_{\nu} / 3$, while for a nonrelativistic ideal gas $P \propto 2 e / 3$. Thus, we expect that their appearance decreases the total pressure and we quantify the relative variation of the pressure to be $\lesssim 0.04$, in particular where the equatorial plane intersects the hot an- nulus outside the merging cores (as visible in the bottom panel of Fig. 10. Based on these results, the internal energy stored in the neutrino field is at most $\sim 8 \%$ of the fluid internal energy.

Effects similar to the ones we have discussed for high density region $\left(\rho \gtrsim 10^{14} \mathrm{~g} \mathrm{~cm}^{-3}\right)$ in the equatorial plane can be seen along any vertical plane passing through the center. However, far from the equatorial plane, the annulus structure involves matter at slightly lower density for which the decrease in the pressure due to the presence of trapped neutrinos is slightly more significant $\left(P_{\text {eq }} / P_{\text {sim }} \sim\right.$ $0.94)$.

We finally move to the analysis of trapped neutrinos in the SFHo_M135135 model. The former is characterized by larger maximum densities and temperatures, see Fig. 1, and by a quick collapse of the central massive NS. Before black hole formation, results of the neutrino post-processing analysis are qualitatively similar to the DD2_M136136 case. Due to the larger temperatures reached during the NS collisions, the proton degeneracy decrease is more pronunced and electron antineutrinos can locally form a trapped gas with $Y_{\bar{\nu}_{e}} \lesssim 0.05$, in particular where the cores collide and at the interface between the spiral arms and the forming disk. The variation of the local pressure to the appearance of neutrinos can reach $10 \%$ of the simulation pressure. However, these large temperatures are reached only immediately after merger, while during most of the evolution and for the bulk of the matter, neutrino fractions are only marginally larger than the ones observed in the DD2_M136136 model. After the formation of an apparent horizon, the maximum density and temperature significantly decrease inside the disk and trapped neutrinos play no role inside the remnant.

\section{Disks around black holes or NS remnant}

The analysis of the entropy distributions inside BNS merger remnants already revealed important differences between the case in which a black hole promptly forms and that in which a massive NS survives for several dynamical time scales (end of Section 3). Further differences in the disk properties are clearly visible in Figs. 11, 13, where we present volume rendering of the matter density, and three dimensional spatial distributions of entropy and electron fraction, for two simulations employing a stiffer (DD2_M136136, left panels) and a softer (SFHo_M135135, right panels) EOS, a few tens of milliseconds after merger.

The disk around the black hole produced at the end of the SFHo_M135135 simulation is less massive and more compact (i.e., with a significantly smaller spatial extension and a steeper density profile) than the disk produced outside the massive NS by the DD2_M136136 simulation. Maximum densities in the former case reach only $\sim 10^{12} \mathrm{~g} \mathrm{~cm}^{-3}$ in a torus around the innermost stable circular orbit. On the other hand, in the latter case the spatially more extended disk has a continuously increasing density profile moving towards the center that joins up with the massive NS density structure. The resulting disk is geometrically and optically thick to neutrinos (at least during the first 
tens of ms). Fig. 12 confirms that a softer EOS produces disks with larger entropies (possibly, $\Delta s \sim 2 k_{\mathrm{B}}$ baryon $^{-1}$ ) due to the stronger shocks that develop at and after merger. Neutrino irradiation from the center of the remnant changes the electron fraction above and inside the disk. On the one hand, in both cases the innermost part of the disk retains its initial neutron richness, while the electron fraction increases significantly above 0.3 in the low density funnel above it. On the other hand, significant differences characterize $Y_{e}$ in the outer parts of the disk. Due to the more extended, more diluite and colder structure, the outer disk obtained in the DD2 run has a lower electron fraction than the more compact disk resulting from the SFHo run.

\section{Symmary and conclusions}

In this paper, we analyzed the thermodynamical conditions experienced by matter during the merger of two NSs based on the results of detailed hydrodynamical simulations in numerical relativity. Our five fiducial models include the effect of finite-temperature, composition dependent nuclear EOSs in tabular form and of the emission of neutrinos via a three-species M0 that accounts for the reabsorption of electron flavor (anti)neutrinos in optically thin conditions.

We explored three different hadronic EOSs: DD2, SFHo and LS220. The first two are based on the RMF approach and on a full distribution of nuclei in NSE, while the latter is based on a liquid droplet Skyrme model and, besides free neutrons and protons, includes $\alpha$ particles and one representative heavy nucleous. While most of our models refer to equal mass binaries, in one case we have investigate the potential impact of an asymmetric mass binary merger. Moreover, in three cases we have included the impact of subgrid-scale turbulent angular momentum transport using the general-relativistic large eddy simulations method (GRLES; 66 ).

Matter stays cold and close to $T=0, \nu$-less weak equilibrium during the inspiral (the marginal increase of temperature at the NS surfaces is a numerical artifact). As soon as the two NS cores touch and merge, the temperature increases by up to several tens of $\mathrm{MeV}$ for densities immediately above nuclear saturation density. This is due to the development of hydrodynamical shockes at the interface between the bouncing cores that produce an hot mantle around the unshocked, merging bulks. The entropy in the cores stays below $2 k_{\mathrm{B}}$ baryon $^{-1}$, while it increases to a few $k_{\mathrm{B}}$ baryon $^{-1}$ in the hot mantle. The action of rotating spiral arms on the forming disk increases temperature and entropy. At first, on a timescale comparable with a few disk dynamical timescale, this produces a large spread in temperature and entropy. Later, over several disk orbits, the conditions becomes more homogeneous. Matter expansion from the merging cores follows $T^{3} / \rho^{2}$ profiles, typical of baryon-dominated matter that expands adiabatically. The more compact and dense NS cores associated with softer EOS produce the largest densities and temperatures. Asymmetric NS masses and the inclusion of turbulent viscosity have only a marginal impact on the thermodynamical properties.

Since our simulations did not include the presence of a trapped neutrino gas in thermal and weak equilibrium with the fluid, we analyzed its potential impact in postprocessing. We concluded that its inclusion can only marginally affect the local thermodynamical properties of dense matter above $10^{12} \mathrm{~g} \mathrm{~cm}^{-3}$ : the maximum temperature is reduced by $\lesssim 10 \%$, while the introduction of neutrinos determines a decrease in the total pressure $\lesssim 5 \%$ and only in the hottest regions of the domain. Due to the initial neutron richness, deviations from cold, $\nu$-less weak equilibrium due to an increase in temperature favor the production of electron antineutrinos over neutrinos.

If according to our results the inclusion of trapped neutrinos is not expected to significantly change the dynamical properties of the remnant, the appearance of additional degrees of freedom such as hyperons and quarks for densities well above $\rho_{0}$ could have a more significant impact. BNS merger simulations performed with the $\mathrm{BHB} \Lambda \phi$ EOS model 94 (which is identical to DD2 apart for the appearance of hyperons at $\rho \gtrsim 2 \rho_{0}$ ) present a qualitatively different evolution of the remnant (see, e.g., 28). Hyperons in the BHB $\Lambda \phi$ models soften the EOS and can lead to a fast collapse of the massive NS into a black hole, whereas identical initial conditions evolved with the DD2 EOS produce a stable rotating NS. For merging NSs with $M_{\mathrm{NS}} \lesssim 1.35 M_{\odot}$ the appearance of hyperons is expected only after merger and mainly inside the cold, unshocked cores. If a black hole does not form, the qualitative behavior of the thermodynamical conditions is expected to be very similar to the DD2 case, even if the stronger core bounces related with the EOS softening produce a hotter remnant in the $\mathrm{BHB} \Lambda \phi$ than in the DD2 case. On the other hand, in more massive NSs hyperons could be present already during the inspiral phase and their fraction increases faster and more significantly during the early post-merger phases. This can lead to hotter remnant disks than the corresponding DD2 cases, if the massive NS collapse to a black hole.

The possible appearance of muons $\left(m_{\mu} \approx 105.6 \mathrm{MeV}\right)$ and mesons (e.g. pions, $m_{\pi} \approx 135-140 \mathrm{MeV}$ ) has been explored, especially in cold NS matter, e.g. 95. In this case, degeneracy drives their production at densities well above $\rho_{0}$. During a BNS merger, if the temperature reaches several tens of $\mathrm{MeV}$ in the hot envelope, they could also be thermally produced at densities around or below $\rho_{0}$. Their inclusion in finite-temperature, composition depedent EOS is still missing and their potential impact on BNS mergers has not been explored yet.

Our fiducial models allowed us to study the properties of disk forming in a BNS merger. We found that the formation of a black hole in the center instead of a massive NS leads to the most significant differences. In the early $\mathrm{BH}$ formation case, the disk is usually hotter (due to the more violent NS collisions), more compact, and less dense $\left(\rho \lesssim 10^{12} \mathrm{~g} \mathrm{~cm}^{-3}\right)$. However, the higher temperatures and neutrino luminosities change its elec- 
tron fraction more significantly. In the case in which a long-lived remnant is formed, the disk is geometrically and optically thick, it is spatially more extended and has less steep density gradients, due to the prolonged action of the non-singular central object. Our post-processing analysis of the trapped-neutrino gas could not directly investigate if the electron fraction of the matter forming the disk is largely affected by the temporal transition to finite-temperature weak equilibrium conditions before quasi-adiabatic expansion. The reduced variation of $Y_{e}$ observed for $\rho \gtrsim 10^{12} \mathrm{~g} \mathrm{~cm}^{-3}$ seems to imply that this is not the case and more significant changes in $Y_{e}$ are expected due to the absorption of electrons and positrons, as weel as of $\nu_{e}$ and $\bar{\nu}_{e}$ on free baryons. This conclusions is qualitatively consistent with results reported by BNS simulations including the contributions of trapped neutrinos, e.g. [75, 76 .

Finally, the histograms of the thermodynamical conditions reported in 87,88 for CCSN simulations allow a direct comparison between the thermodynamical conditions inside CCSNe and BNS mergers. The electron fraction has opposite trends inside these two scenarios. In CCSNe, symmetric nuclear matter deleptonizes to reach neutron rich conditions inside the forming proto NS. Cold, $\nu$-less weak equilibrium conditions are achieved only on the $\nu$ cooling timescale (much larger than the dynamical and explosion timescale). On the other hand, in BNS mergers the decompression, heating, and neutrino irradiation of cold nuclear matter drive matter leptonization. This process is relevant to set the thermodynamical conditions of the ejecta and, ultimately, its nucleosynthesis yields, e.g [90, 96, 97, 75, 72, 98, 99. This fundamental difference reflects also on the evolution of the density-temperature conditions. In CCSN simulations the presence of a single shock front between the proto NS and the cold accreting flow channels most of the hot mantle in a rather narrow band in the $\rho-T$ plane. On the other hand, in BNS mergers the combined presence of hydrodynamical compression, tidal decompression, shocks and expansion produces a much broader band that narrows over several dynamical timescales. In CCSNe larger densities and temperatures are achieved for softer EOSs, but the mass of the progenitor star is the most crucial parameter that influences, for example, the maximum temperature reached during the simulation. Indeed, densities of the order of a few $\rho_{0}$ are reached only after the proto NS formation and cooling, and the details of the nuclear interactions are of secondary importance with respect to the strength of the gravitational field. In this work, we have only partially explored the dependence on the colliding NS masses and, in particular, we did not consider very massive NSs. However, the densities are significantly larger in NS mergers and the details of the nuclear interactions influence more deeply the dynamics. Thus, the variability introduced by the different EOSs in NS merger models is compatible with the variability associated with different progenitors for CCSNe. We might expect more variability in mergers with larger NS masses, however prompt collapse to black hole sets an intrinsic limit to the densities reached in the remnant.
Acknowledgments The authors thank Domenico Logoteta for useful discussions. The authors thank the organizers and participants of the INT Program INT-18-72R "First Multi-Messenger Observation of a Neutron Star Merger and its Implications for Nuclear Physics INT workshop" held at (Seattle, March 2018), of the ExtreMe Matter Institute's rapid task force meeting at GSI/FAIR (Darmstadt, June 2018), of the GWEOS workshop (Pisa, February 2019), for stimulating discussions. SB acknowledges support by the EU H2020 under ERC Starting Grant, no. BinGraSp-714626. DR acknowledges support from a Frank and Peggy Taplin Membership at the Institute for Advanced Study and the Max-Planck/Princeton Center (MPPC) for Plasma Physics (NSF PHY-1804048). Computations were performed on the supercomputer SuperMUC at the LRZ Munich (Gauss project pn56zo), on supercomputer Marconi at CINECA (ISCRA-B project number HP10B2PL6K and HP10BMHFQQ); on the supercomputers Bridges, Comet, and Stampede (NSF XSEDE allocation TG-PHY160025); on NSF/NCSA Blue Waters (NSF AWD-1811236).

\section{References}

1. M. Shibata, K. Uryu, Phys. Rev. D61, 064001 (2000), gr-qc/9911058

2. Y. Sekiguchi, K. Kiuchi, K. Kyutoku, M. Shibata, Phys.Rev.Lett. 107, 211101 (2011), 1110.4442

3. S. Bernuzzi, D. Radice, C.D. Ott, L.F. Roberts, P. Moesta, F. Galeazzi, Phys. Rev. D94, 024023 (2016), 1512.06397

4. D. Radice, A. Perego, K. Hotokezaka, S.A. Fromm, S. Bernuzzi, L.F. Roberts, Astrophys. J. 869, 130 (2018), 1809. 11161

5. L.F. Roberts, G. Shen, V. Cirigliano, J.A. Pons, S. Reddy, S.E. Woosley, Physical Review Letters 108, 061103 (2012), 1112.0335

6. J.M. Lattimer, Ann. Rev. Nucl. Part. Sci. 62, 485 (2012), 1305.3510

7. F. zel, P. Freire, Ann. Rev. Astron. Astrophys. 54, 401 (2016), 1603.02698

8. M. Oertel, M. Hempel, T. Klhn, S. Typel, Rev. Mod. Phys. 89, 015007 (2017), 1610.03361

9. J.M. Lattimer, F.D. Swesty, Nucl. Phys. A535, 331 (1991)

10. H. Shen, H. Toki, K. Oyamatsu, K. Sumiyoshi, Nucl. Phys. A637, 435 (1998), nucl-th/9805035

11. I. Bombaci, D. Logoteta, Astron. Astrophys. 609, A128 (2018), 1805.11846

12. T. Damour, Gravitational radiation and the motion of compact bodi in Gravitational Radiation, edited by N. Deruelle, T. Piran (North-Holland, Amsterdam, 1983), pp. 59-144

13. E.E. Flanagan, T. Hinderer, Phys.Rev. D77, 021502 (2008), 0709.1915

14. T. Hinderer, B.D. Lackey, R.N. Lang, J.S. Read, Phys. Rev. D81, 123016 (2010), 0911.3535

15. T. Damour, A. Nagar, Phys. Rev. D81, 084016 (2010), 0911.5041

16. T. Damour, A. Nagar, L. Villain, Phys.Rev. D85, 123007 (2012), 1203.4352

17. S. Bernuzzi, A. Nagar, M. Thierfelder, B. Brügmann, Phys.Rev. D86, 044030 (2012), 1205.3403 
18. S. Bernuzzi, A. Nagar, S. Balmelli, T. Dietrich, M. Ujevic, Phys.Rev.Lett. 112, 201101 (2014), 1402.6244

19. F. Zappa, S. Bernuzzi, D. Radice, A. Perego, T. Dietrich, Phys. Rev. Lett. 120, 111101 (2018), 1712.04267

20. K. Hotokezaka, K. Kyutoku, H. Okawa, M. Shibata, K. Kiuchi, Phys.Rev. D83, 124008 (2011), 1105.4370

21. A. Bauswein, T. Baumgarte, H.T. Janka, Phys.Rev.Lett. 111, 131101 (2013), 1307.5191

22. D. Radice, A. Perego, F. Zappa, S. Bernuzzi, Astrophys. J. 852, L29 (2018), 1711.03647

23. D. Radice, A. Perego, S. Bernuzzi, B. Zhang, Mon. Not. Roy. Astron. Soc. 481, 3670 (2018), 1803.10865

24. D. Eichler, M. Livio, T. Piran, D.N. Schramm, Nature 340 , $126(1989)$

25. E. Nakar, Phys. Rept. 442, 166 (2007), astro-ph/0701748

26. S. Rosswog, Int.J.Mod.Phys. D24, 1530012 (2015), 1501.02081

27. O. Just, M. Obergaulinger, H.T. Janka, A. Bauswein, N. Schwarz, Astrophys. J. 816, L30 (2016), 1510.04288

28. D. Radice, S. Bernuzzi, W. Del Pozzo, L.F. Roberts, C.D. Ott, Astrophys. J. 842, L10 (2017), 1612.06429

29. E.R. Most, L.J. Papenfort, V. Dexheimer, M. Hanauske, S. Schramm, H. Stcker, L. Rezzolla (2018), 1807.03684

30. A. Bauswein, N.U.F. Bastian, D.B. Blaschke, K. Chatziioannou, J.A. Clark, T. Fischer, M. Oertel, Phys. Rev. Lett. 122, 061102 (2019), 1809.01116

31. B.P. Abbott et al. (Virgo, LIGO Scientific), Phys. Rev. Lett. 119, 161101 (2017), 1710.05832

32. B.P. Abbott et al. (LIGO Scientific, Virgo), Phys. Rev. X9, 011001 (2019), 1805.11579

33. B.P. Abbott et al. (LIGO Scientific, Virgo), Phys. Rev. Lett. 121, 161101 (2018), 1805.11581

34. S. De, D. Finstad, J.M. Lattimer, D.A. Brown, E. Berger, C.M. Biwer (2018), 1804.08583

35. L. Baiotti, T. Damour, B. Giacomazzo, A. Nagar, L. Rezzolla, Phys. Rev. D84, 024017 (2011), 1103.3874

36. D. Radice, L. Rezzolla, F. Galeazzi, Mon.Not.Roy.Astron.Soc. 437, L46 (2014), 1306.6052

37. K. Hotokezaka, K. Kyutoku, H. Okawa, M. Shibata, Phys. Rev. D91, 064060 (2015), 1502.03457

38. A. Nagar et al., Phys. Rev. D98, 104052 (2018), 1806.01772

39. B.P. Abbott et al. (Virgo, LIGO Scientific), Astrophys. J 851, L16 (2017), 1710.09320

40. A. Bauswein, H.T. Janka, Phys.Rev.Lett. 108, 011101 (2012), 1106.1616

41. K. Takami, L. Rezzolla, L. Baiotti, Phys.Rev.Lett. 113 , 091104 (2014), 1403.5672

42. S. Bernuzzi, T. Dietrich, A. Nagar, Phys. Rev. Lett. 115, 091101 (2015), 1504.01764

43. H. Yang, V. Paschalidis, K. Yagi, L. Lehner, F. Pretorius, N. Yunes (2017), 1707.00207

44. K. Chatziioannou, J.A. Clark, A. Bauswein, M. Millhouse, T.B. Littenberg, N. Cornish, Phys. Rev. D96, 124035 (2017), 1711.00040

45. B. Margalit, B.D. Metzger, Astrophys. J. 850, L19 (2017), 1710.05938

46. M. Shibata, S. Fujibayashi, K. Hotokezaka, K. Kiuchi, K. Kyutoku, Y. Sekiguchi, M. Tanaka, Phys. Rev. D96, 123012 (2017), 1710.07579

47. L. Rezzolla, E.R. Most, L.R. Weih, Astrophys. J. 852, L25 (2018), 1711.00314
48. M. Ruiz, S.L. Shapiro, A. Tsokaros, Phys. Rev. D97, 021501 (2018), 1711.00473

49. A. Bauswein, O. Just, H.T. Janka, N. Stergioulas, Astrophys. J. 850, L34 (2017), 1710.06843

50. D. Radice, L. Dai (2018), 1810.12917

51. P. Demorest, T. Pennucci, S. Ransom, M. Roberts, J. Hessels, Nature 467, 1081 (2010), 1010.5788

52. J. Antoniadis, P.C. Freire, N. Wex, T.M. Tauris, R.S Lynch et al., Science 340, 6131 (2013), 1304.6875

53. S. Typel, G. Ropke, T. Klahn, D. Blaschke, H.H. Wolter, Phys. Rev. C81, 015803 (2010), 0908.2344

54. M. Hempel, J. Schaffner-Bielich, Nucl. Phys. A837, 210 (2010), 0911.4073

55. A.W. Steiner, M. Hempel, T. Fischer, Astrophys. J. 774, 17 (2013), 1207.2184

56. Eric Gourgoulhon, Philippe Grandclément, Jean-Alain Marck, Jérôme Novak and Keisuke Taniguchi, http:// www.lorene.obspm.fr/, Paris Observatory, Meudon section - LUTH laboratory

57. S. Bernuzzi, D. Hilditch, Phys. Rev. D81, 084003 (2010), 0912.2920

58. D. Hilditch, S. Bernuzzi, M. Thierfelder, Z. Cao, W. Tichy et al., Phys. Rev. D88, 084057 (2013), 1212.2901

59. D. Radice, L. Rezzolla, Astron. Astrophys. 547, A26 (2012), 1206.6502

60. D. Radice, L. Rezzolla, F. Galeazzi, Class.Quant.Grav. 31, 075012 (2014), 1312.5004

61. D. Radice, L. Rezzolla, F. Galeazzi, ASP Conf. Ser. 498, 121 (2015), 1502.00551

62. M.J. Berger, J. Oliger, J.Comput.Phys. 53, 484 (1984)

63. M.J. Berger, P. Colella, Journal of Computational Physics 82, 64 (1989)

64. C. Reisswig, R. Haas, C.D. Ott, E. Abdikamalov, P. Msta, D. Pollney, E. Schnetter, Phys. Rev. D87, 064023 (2013), 1212.1191

65. E. Schnetter, S.H. Hawley, I. Hawke, Class.Quant.Grav. 21, 1465 (2004), gr-qc/0310042

66. D. Radice, Astrophys. J. 838, L2 (2017), 1703.02046

67. N.I. Shakura, R.A. Sunyaev, Astron. Astrophys. 24, 337 (1973)

68. K. Kiuchi, K. Kyutoku, Y. Sekiguchi, M. Shibata, Phys. Rev. D97, 124039 (2018), 1710.01311

69. S. Rosswog, M. Liebendoerfer, Mon.Not.Roy.Astron.Soc. 342, 673 (2003), astro-ph/0302301

70. N. Itoh, H. Hayashi, A. Nishikawa, Y. Kohyama, Astrophys. J. Suppl. 102, 411 (1996)

71. F. Galeazzi, W. Kastaun, L. Rezzolla, J.A. Font, Phys.Rev. D88, 064009 (2013), 1306.4953

72. D. Radice, F. Galeazzi, J. Lippuner, L.F. Roberts, C.D. Ott, L. Rezzolla, Mon. Not. Roy. Astron. Soc. 460, 3255 (2016), 1601.02426

73. D. Neilsen, S.L. Liebling, M. Anderson, L. Lehner, E. OConnor et al., Phys.Rev. D89, 104029 (2014), 1403.3680

74. M.H. Ruffert, H.T. Janka, G. Schäfer, Astron. Astrophys. 311, 532 (1996), astro-ph/9509006

75. Y. Sekiguchi, K. Kiuchi, K. Kyutoku, M. Shibata, Phys.Rev. D91, 064059 (2015), 1502.06660

76. F. Foucart, E. O'Connor, L. Roberts, M.D. Duez, R. Haas, L.E. Kidder, C.D. Ott, H.P. Pfeiffer, M.A. Scheel, B. Szilagyi, Phys. Rev. D91, 124021 (2015), 1502.04146

77. F. Foucart, M.D. Duez, L.E. Kidder, R. Nguyen, H.P. Pfeiffer, M.A. Scheel (2018), 1806.02349 
78. S.W. Bruenn, Astrophys. J. Suppl. 58, 771 (1985)

79. A. Burrows, S. Reddy, T.A. Thompson, Nucl. Phys. A777, 356 (2006), astro-ph/0404432

80. S.L. Shapiro, S.A. Teukolsky, Black holes, white dwarfs, and neutron stars: The physics of compact objects (Wiley, New York, USA, 1983)

81. A. Perego, R. Cabezon, R. Kaeppeli, Astrophys. J. Suppl. 223, 22 (2016), 1511.08519

82. J. Kaplan, C. Ott, E. O'Connor, K. Kiuchi, L. Roberts et al., Astrophys.J. 790, 19 (2014), 1306.4034

83. K. Hotokezaka, K. Kiuchi, K. Kyutoku, H. Okawa, Y.i. Sekiguchi et al., Phys.Rev. D87, 024001 (2013), 1212.0905

84. A. Bauswein, S. Goriely, H.T. Janka, Astrophys.J. 773, 78 (2013), 1302.6530

85. N. Stergioulas, A. Bauswein, K. Zagkouris, H.T. Janka, Mon.Not.Roy.Astron.Soc. 418, 427 (2011), 1105.0368

86. S. Bernuzzi, T. Dietrich, W. Tichy, B. Brügmann, Phys.Rev. D89, 104021 (2014), 1311.4443

87. S. Bacca, K. Hally, M. Liebendorfer, A. Perego, C.J. Pethick, A. Schwenk, Astrophys. J. 758, 34 (2012), 1112.5185

88. T. Fischer, M. Hempel, I. Sagert, Y. Suwa, J. SchaffnerBielich, Eur. Phys. J. A50, 46 (2014), 1307.6190

89. S. Lalit, M.A.A. Mamun, C. Constantinou, M. Prakash, Eur. Phys. J. A55, 10 (2019), 1809.08126

90. A. Perego, S. Rosswog, R. Cabezon, O. Korobkin, R. Kaeppeli et al., Mon.Not.Roy.Astron.Soc. 443, 3134 (2014), 1405.6730

91. W. Kastaun, R. Ciolfi, A. Endrizzi, B. Giacomazzo, Phys. Rev. D96, 043019 (2017), 1612.03671

92. J.P. Cox, R.T. Giuli, Principles of stellar structure (Gordon and Breach, New York, 1968)

93. K. Takahashi, M.F. El Eid, W. Hillebrandt, Astron.Astrophys. 67, 185 (1978)

94. S. Banik, M. Hempel, D. Bandyopadhyay, Astrophys. J. Suppl. 214, 22 (2014), 1404.6173

95. N.K. Glendenning, Compact Stars (Springer-Verlag, New York, 1996)

96. B.D. Metzger, R. Fernndez, Mon.Not.Roy.Astron.Soc. 441, 3444 (2014), 1402.4803

97. O. Just, A. Bauswein, R.A. Pulpillo, S. Goriely, H.T. Janka, Mon. Not. Roy. Astron. Soc. 448, 541 (2015), 1406.2687

98. F. Foucart, E. O'Connor, L. Roberts, L.E. Kidder, H.P. Pfeiffer, M.A. Scheel, Phys. Rev. D94, 123016 (2016), 1607.07450

99. J. Lippuner, R. Fernndez, L.F. Roberts, F. Foucart, D. Kasen, B.D. Metzger, C.D. Ott, Mon. Not. Roy. Astron. Soc. 472, 904 (2017), 1703.06216 

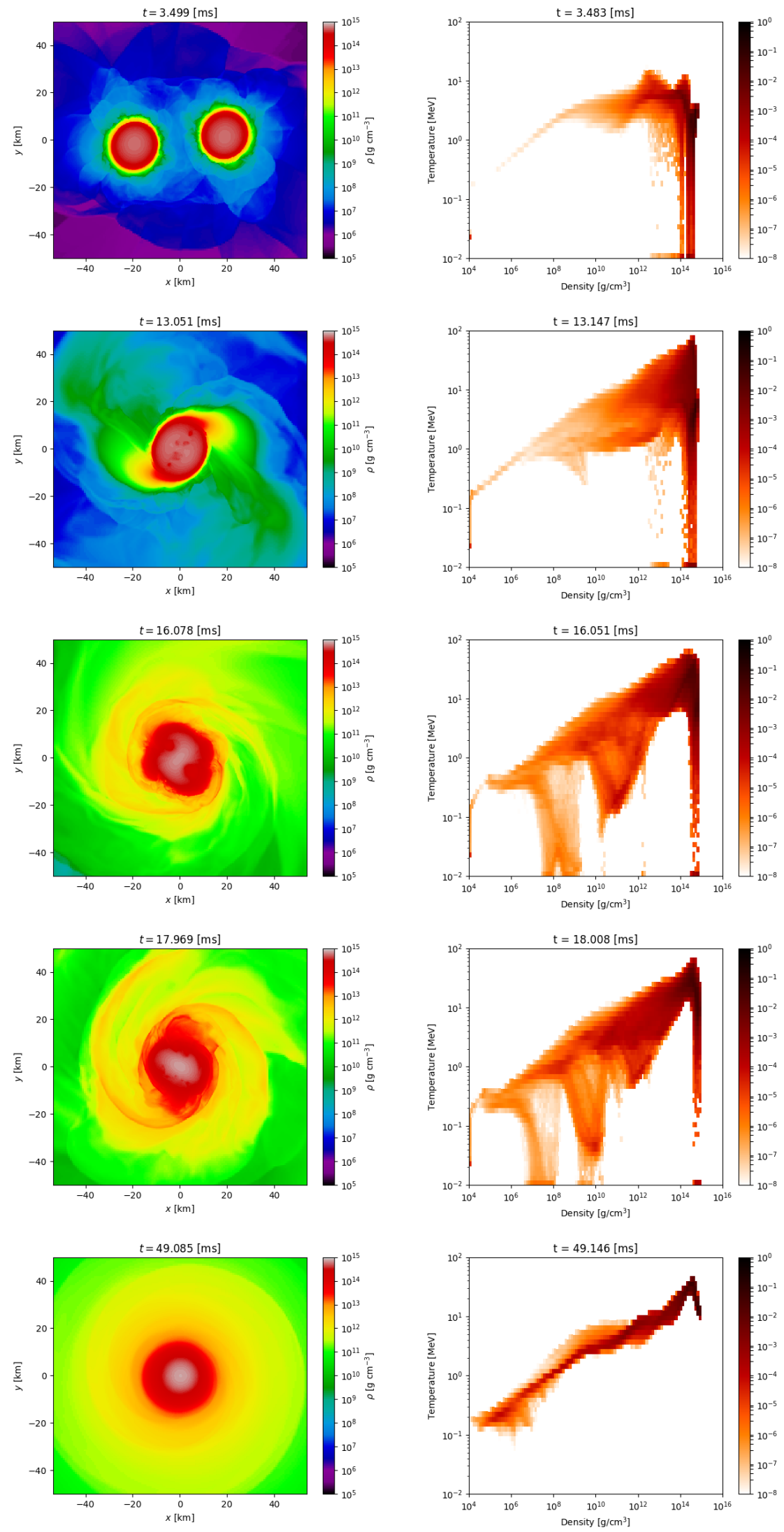
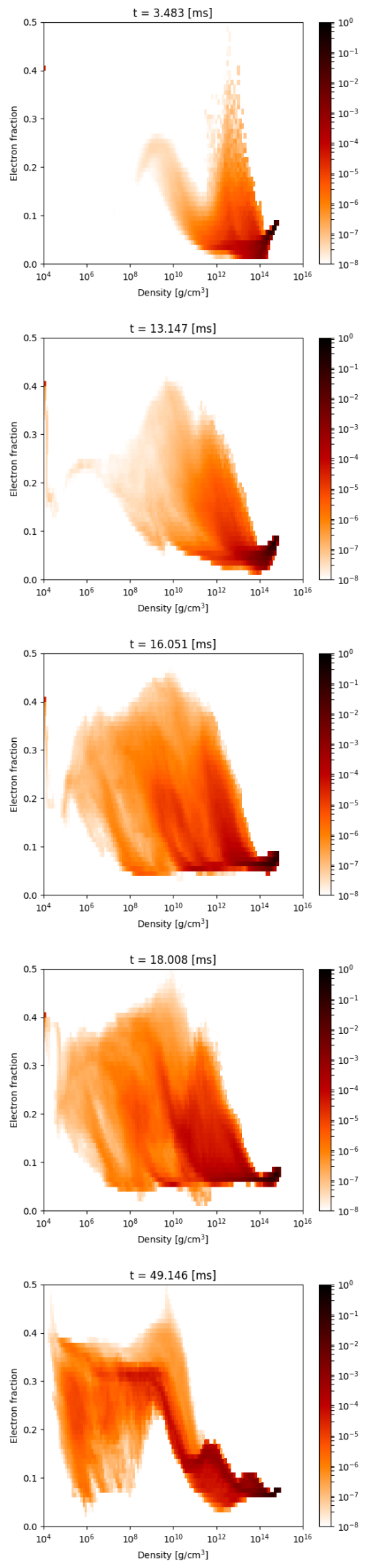

Fig. 2. Rest-mass density on the orbital plane (left), and corresponding histograms on the density-temperature (middle) and density-electron fraction (right) planes for the simulation DD2_M136136. The five rows correspond to the different phases of the merger; moving from the top to the bottom: inspiral phase, time of the temperature peak, 3-4 ms after the temperature peak, time close to the massive NS collapse (if present), end of the simulation. The time reported above each panel refers to the time from the beginning of the simulation in ms. Note $2 \mathrm{D}$ snapshot do not exactly correspond to same times. 

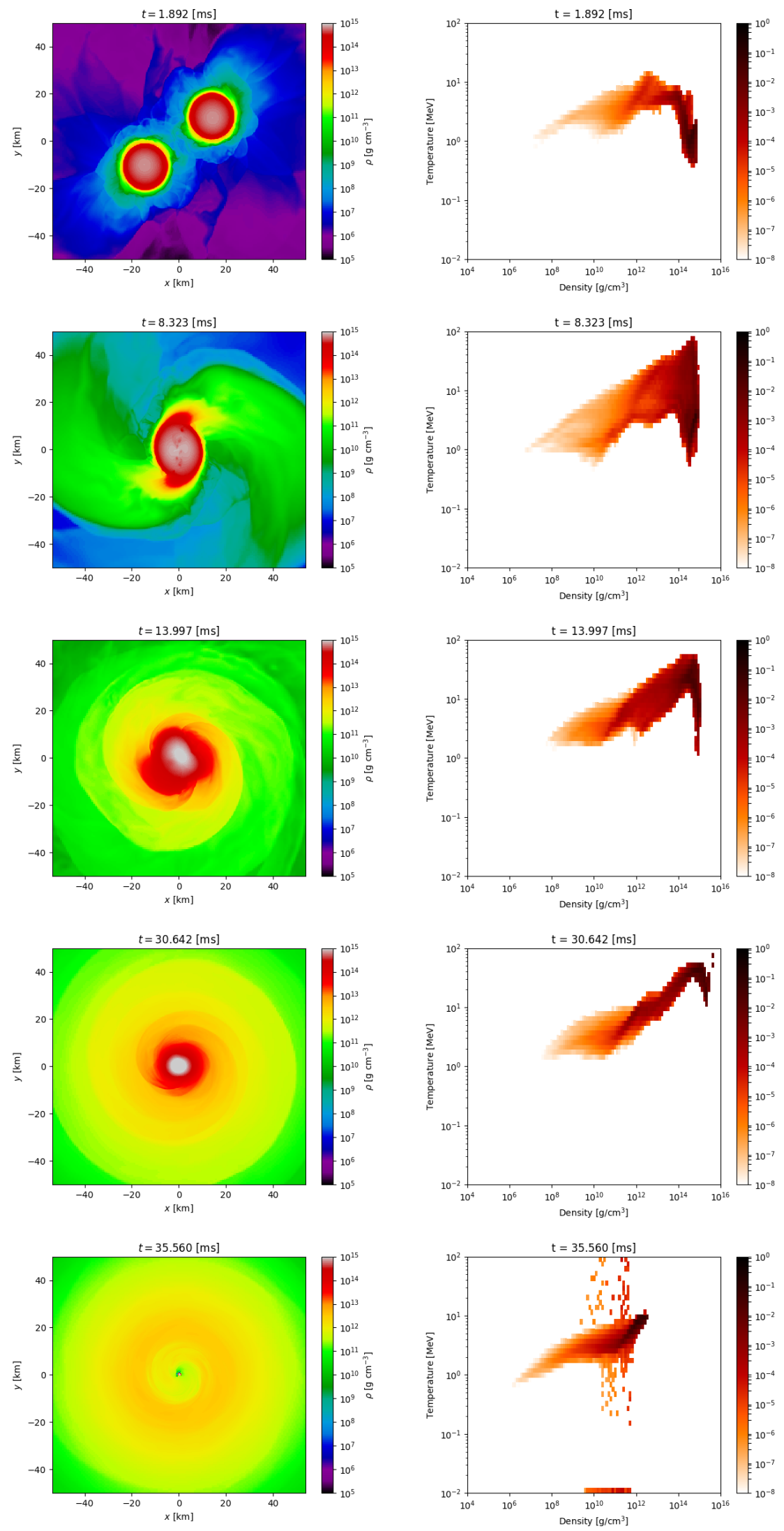
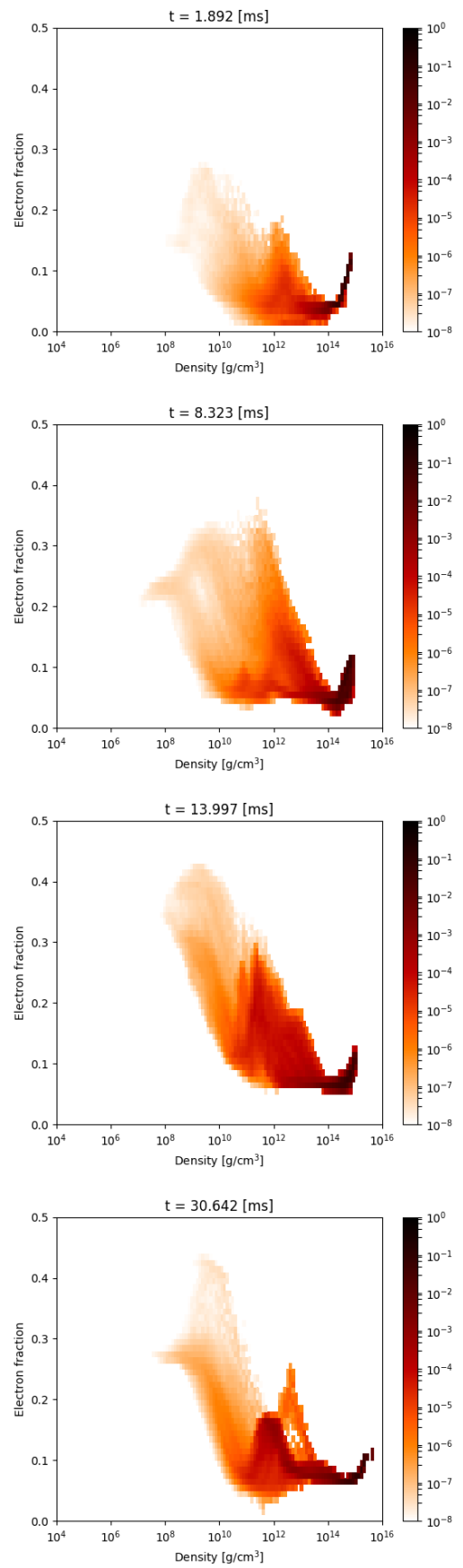

Fig. 3. Same as in Fig. 2, but for simulation LS220_M135135. 

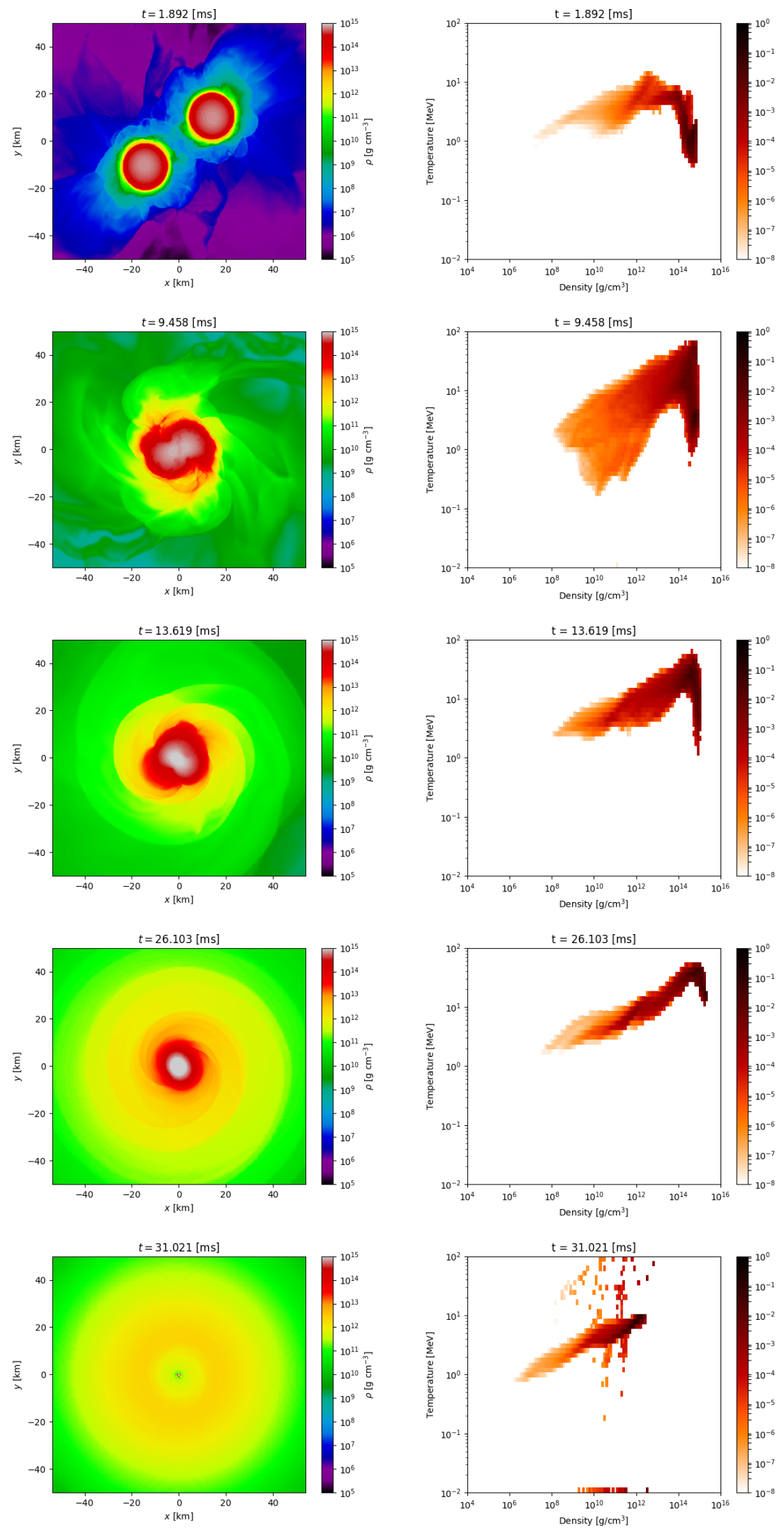
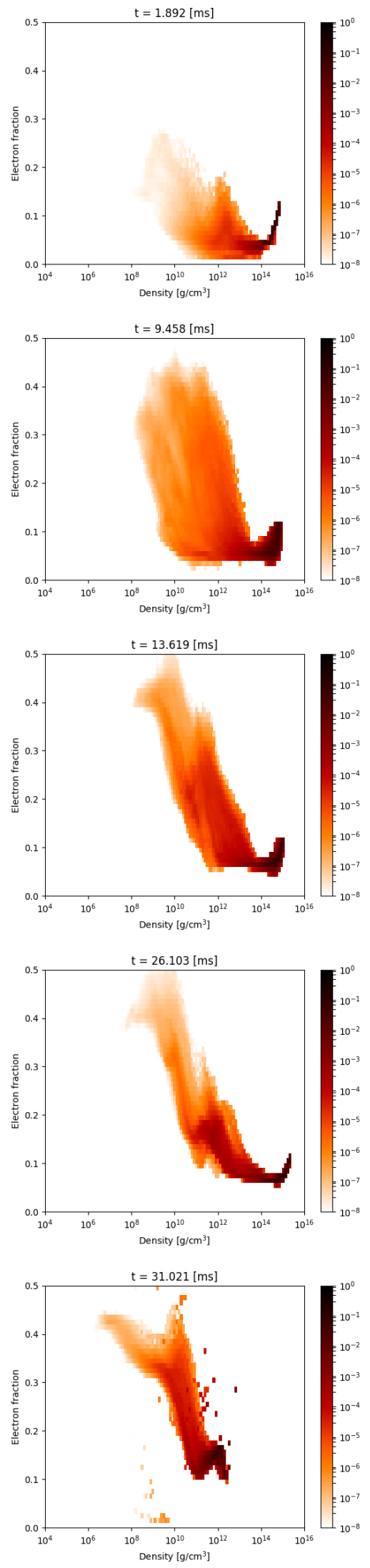

Fig. 4. Same as in Fig. 2, but for simulation LS220_M135135V. 

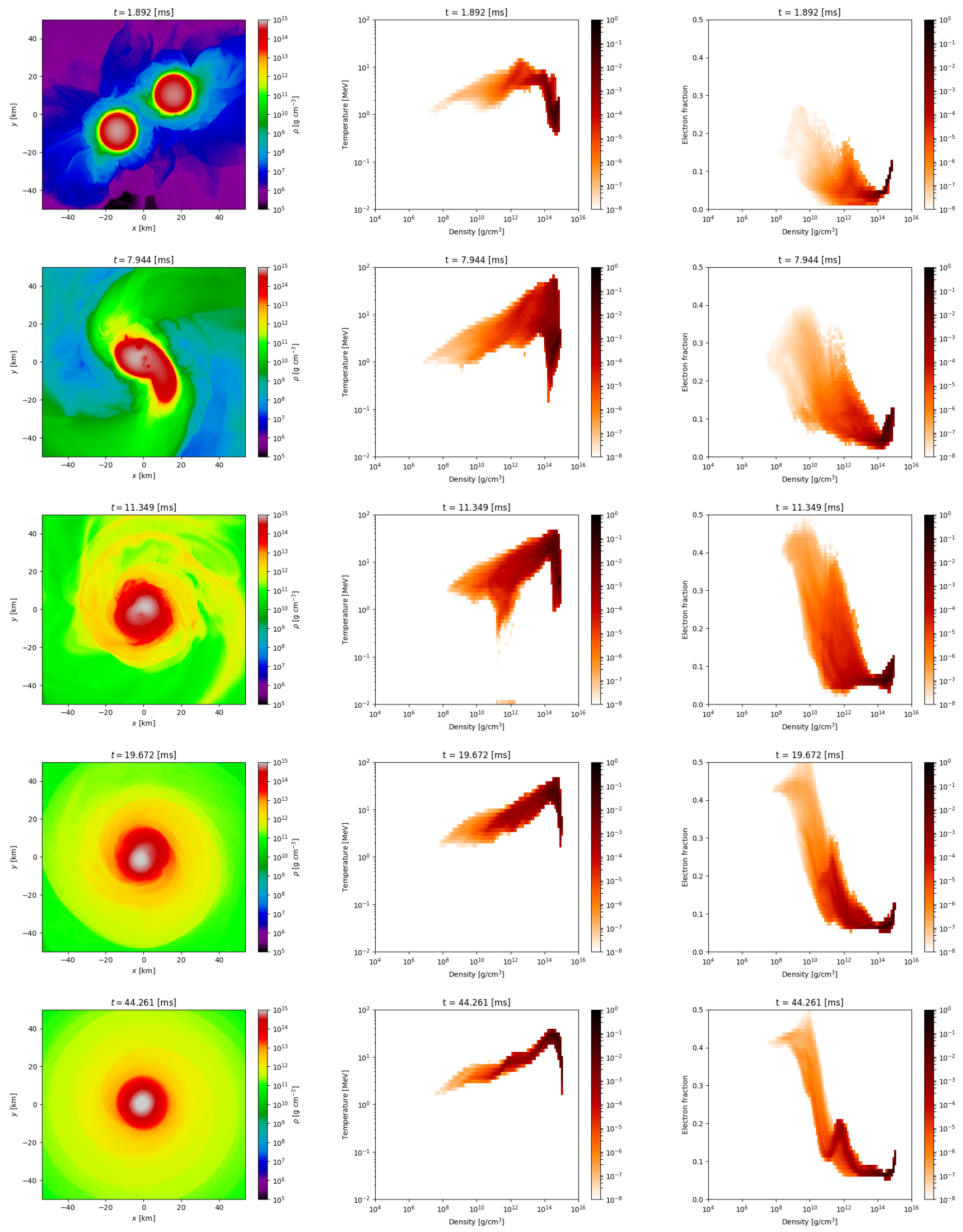

Fig. 5. Same as in Fig. 2] but for simulation LS220_M140120V. 

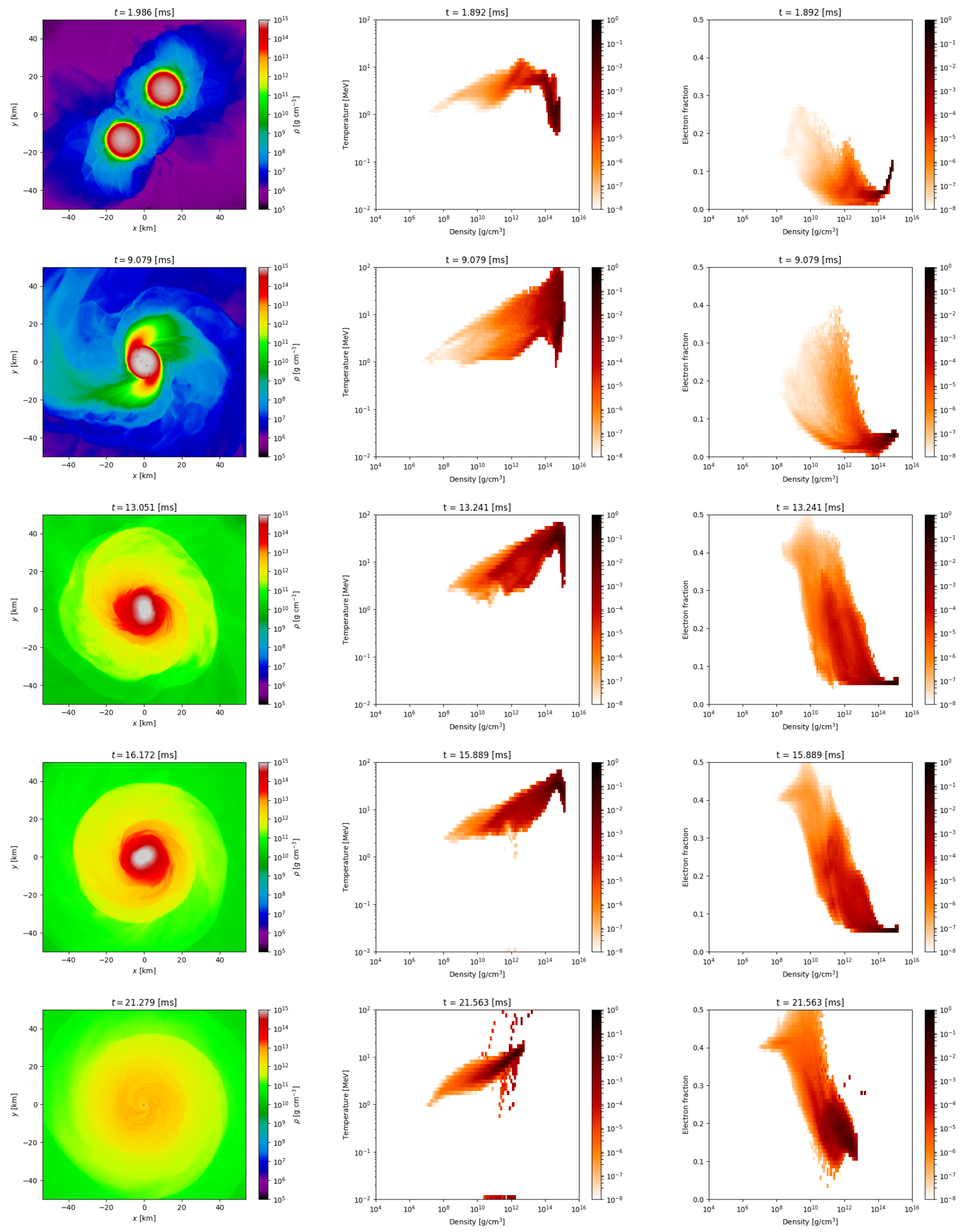

Fig. 6. Same as in Fig. 2 but for simulation SFHo_M135135. 

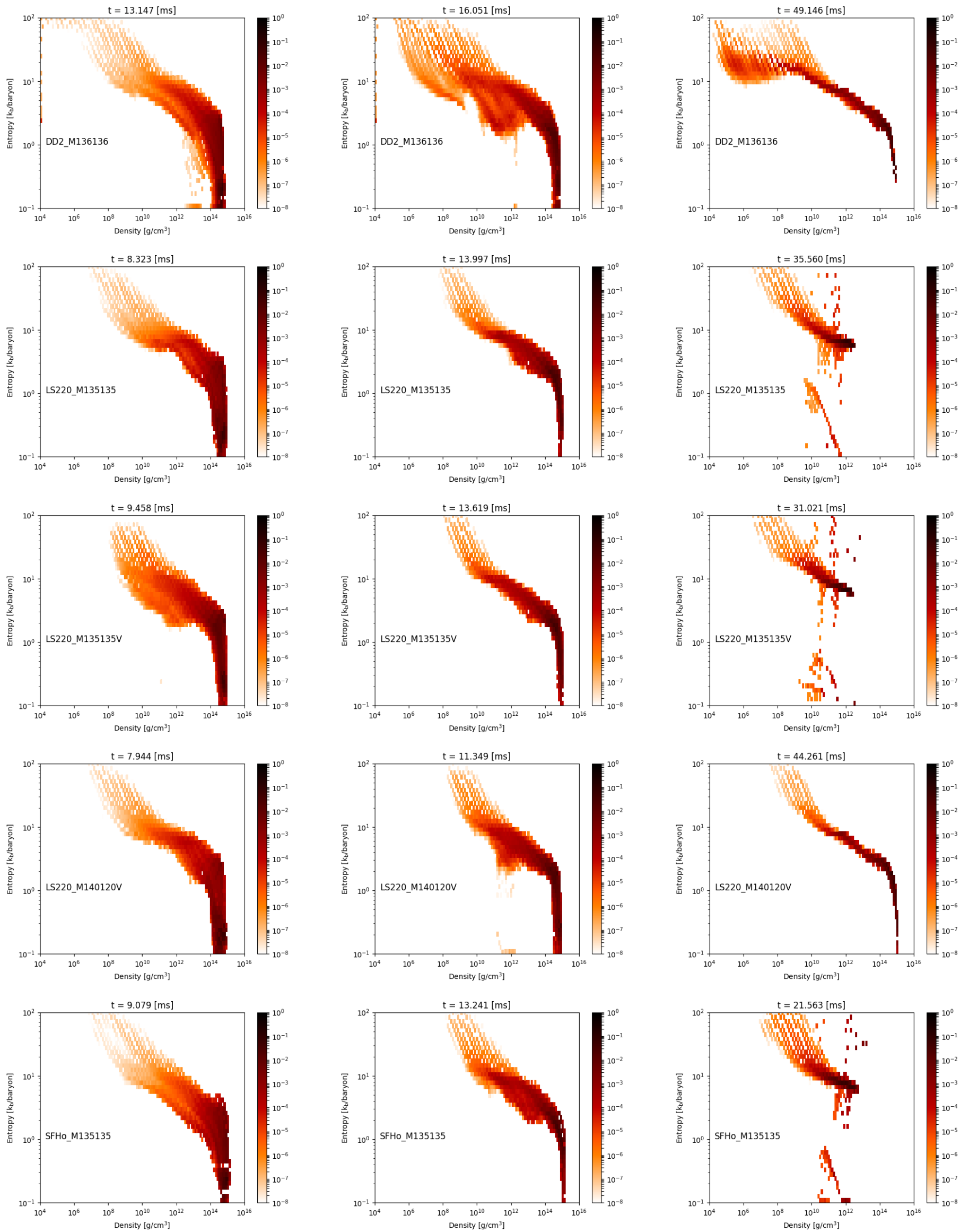

Fig. 7. Histograms of the baryonic mass in the density-entropy plane from the second (left), third (middle) and fifth (right) snapshots for all the five reference simulations reported in Table 2 and in Figs. 2.6. The unshocked cores of the NSs retain their initial low entropy, while shocked decompressed matter expanding towards lower densities experience a significant entropy increase. 

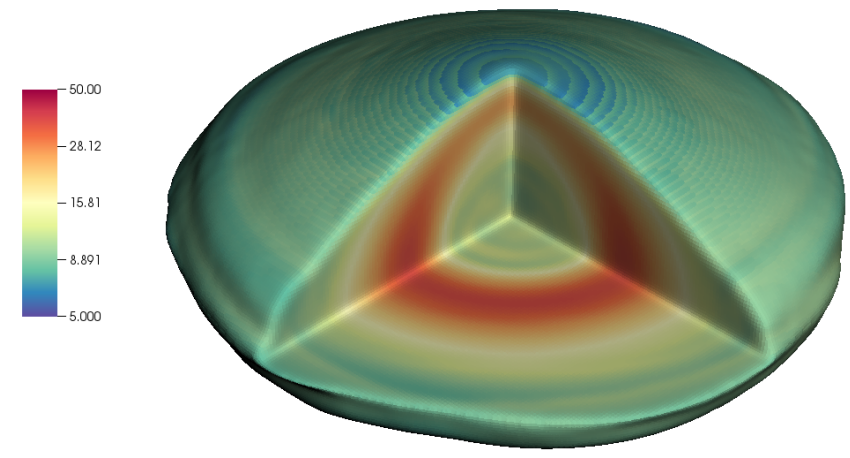

Fig. 8. Temperature distribution $(\mathrm{MeV})$ in the massive NS produced by the DD2_M1361364 binary at $t=34.2 \mathrm{~ms}$ after the merger. The surface of the massive NS is defined as the point where the rest mass density $\rho$ exceeds $10^{13} \mathrm{~g} \mathrm{~cm}^{-3}$. Material at lower densities is made transparent in this visualization. Massive NSs produced in binary mergers are rotationally compressed along the rotation axis (vertically in the figure). High temperatures are confined to a spherical annulus at intermediate densities $\sim 10^{14} \mathrm{~g} \mathrm{~cm}^{-3}$ formed of material originally at the collisional interface between the NSs. 

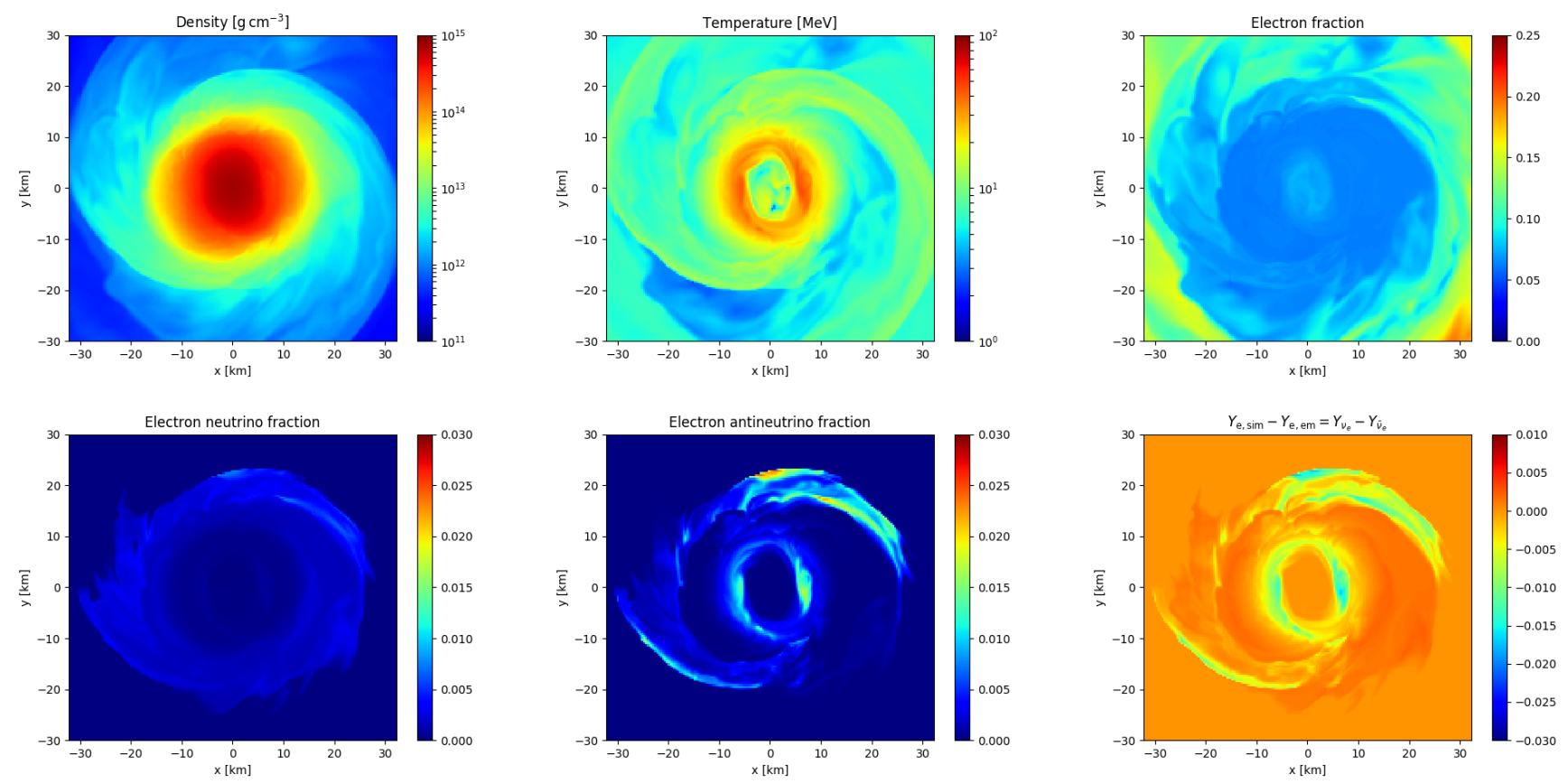

Fig. 9. Upper row: Rest mass density (left), temperature (middle) and electron fraction (right) on the orbital plane for the DD2_M136136 model at the temperature peak, as obtained in simulation. Bottom row: electron neutrino fraction ( $Y_{\nu_{e}}$, left), electron antineutrino fraction $\left(Y_{\bar{\nu}_{e}}\right.$, middle), variation of the electron fraction $\left(Y_{e, \text { sim }}-Y_{e, \text { eq }}\right.$ corresponding to $Y_{\nu_{e}}-Y_{\bar{\nu}_{e}}$, right) after modelling in postoprocessing the presence of trapped neutrinos at high density. Inside the merging cores, matter degeneracy prevents neutrino formation $\left(T_{F} \gg T\right)$. Only for shocked matter in semi-degenerate conditions engulfing the cores or developing at spiral arm interfaces, a significant $\bar{\nu}_{e}$ fractions develops. 

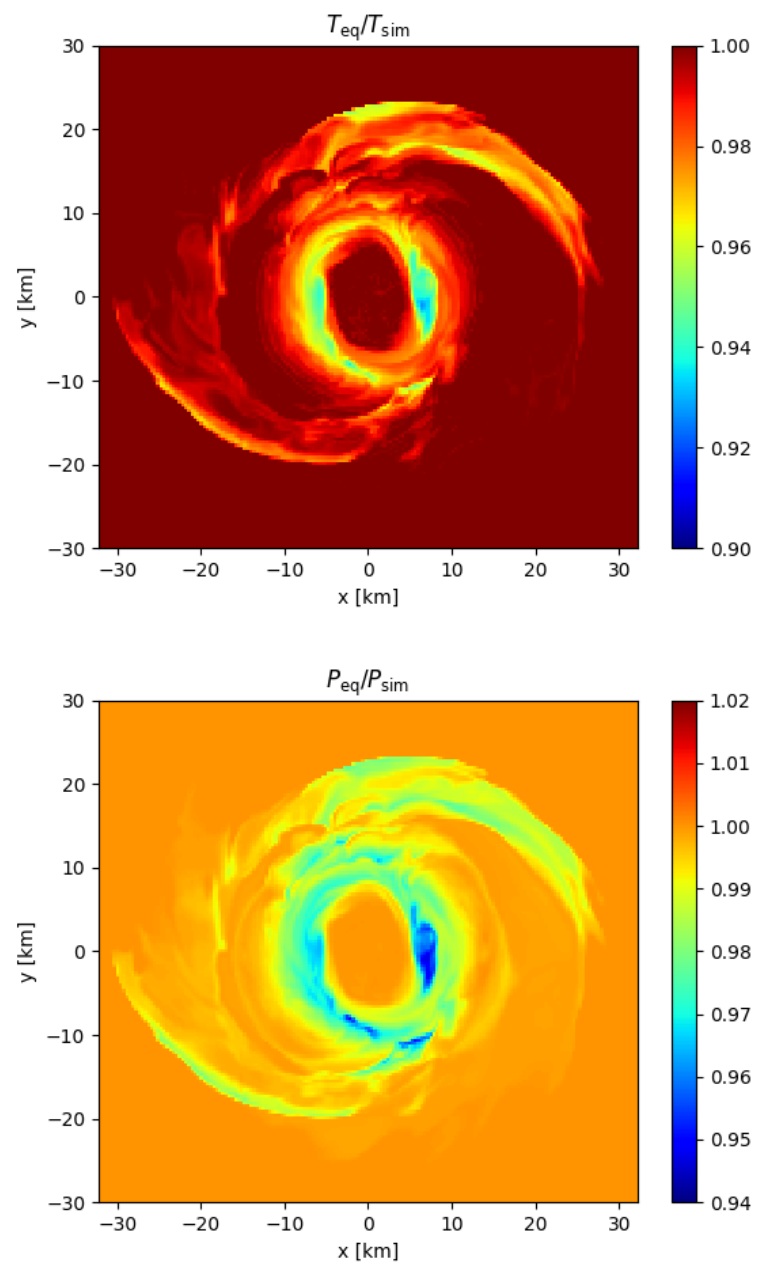

Fig. 10. Upper (lower) panel: ratio of equilibrium temperature (pressure) to the one obtained in the simulation. The equilibrium values are calculated in postprocessing including the effect of trapped neutrinos. The formation of trapped neutrino gases converts fluid thermal energy into radiation energy, reducing matter temperature down to $\sim 92 \%$ of the simulation value. Since $P_{\nu} \propto Z_{\nu} / 3$, the total pressure reduces down to $\sim 94 \%$ of its original value. 

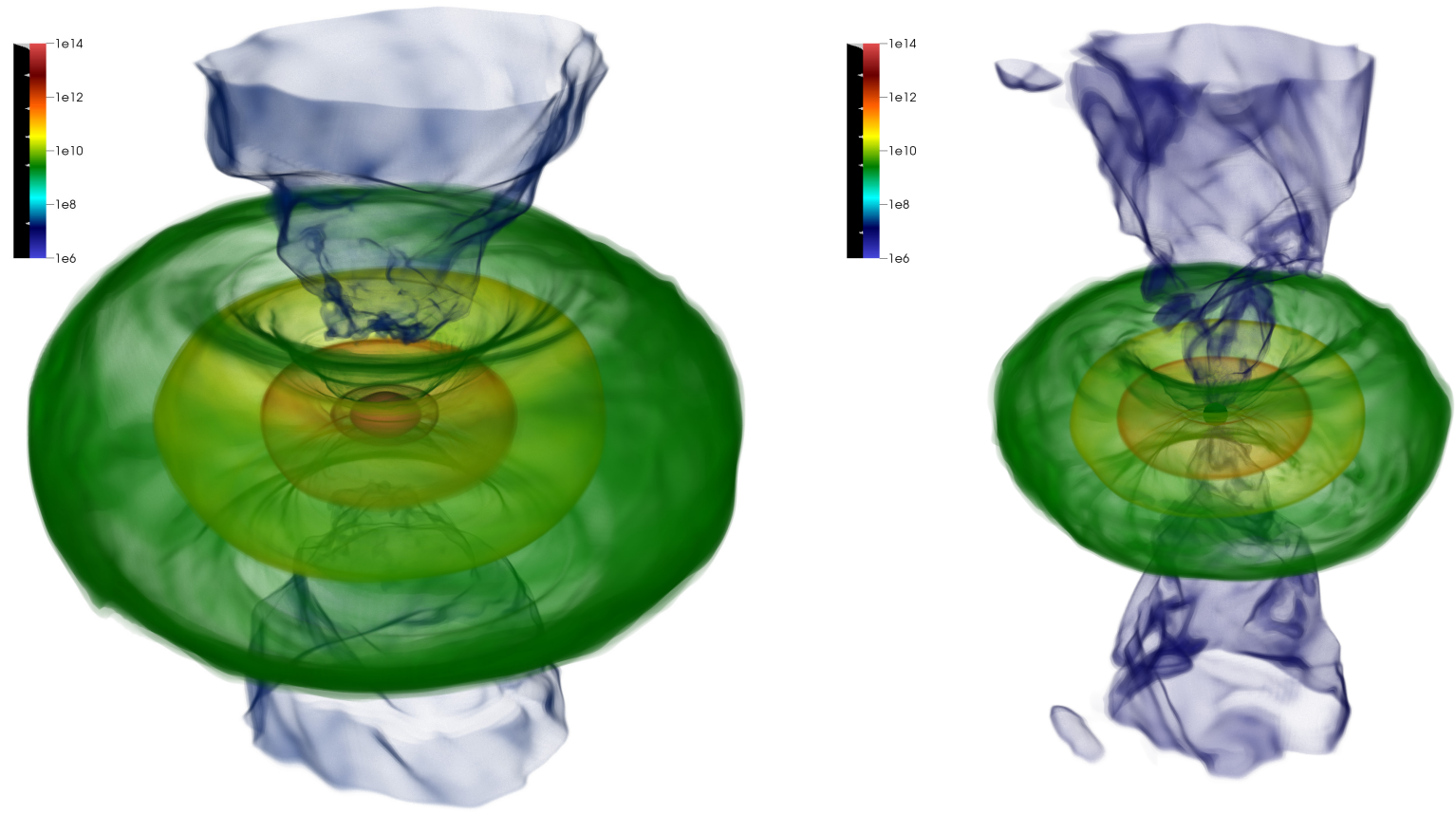

Fig. 11. Volume rendering of the density of the postmerger remnant. Left panel: DD2_M13641364 at $t=34.2 \mathrm{~ms}$ after the merger. Right panel: SFHo_M135135 at $t=17.2 \mathrm{~ms}$ after the merger. Both image have the same spatial scale and show the data in a box of size $\sim 750 \mathrm{~km}$. The black surface denotes the approximate location of the black hole horizon, which we identify as $\alpha=0.3$, being $\alpha$ the lapse function. Remnants harboring black holes at their centers, such as SFHo_M135135, produce smaller, compact disks, that are mostly transparent to neutrinos. Conversely, remnants having a massive NS at their center, have more massive, geometrically and optically thick disks. At high latitudes, a low-density funnel is present in both cases.
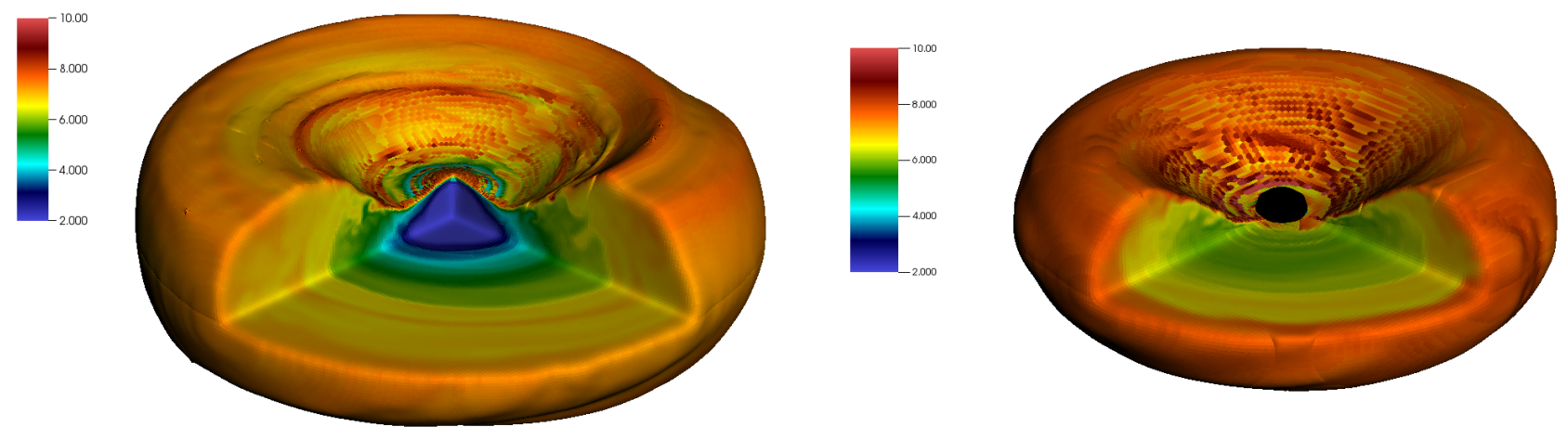

Fig. 12. Entropy in $k_{\mathrm{B}}$ per baryon of the postmerger remnant. Left panel: DD2_M136136 at $t=34.2 \mathrm{~ms}$ after the merger. Right panel: SFHo_M135135 at $t=17.2 \mathrm{~ms}$ after the merger. We only show material with $\rho \geq 10^{11} \mathrm{~g} \mathrm{~cm}^{-3}$ corresponding the inner regions of Fig. 11 Note that here we are using two different spatial scales for the two images (c.f., Fig. 11). The black surface denotes the approximate location of the black hole horizon, which we identify as $\alpha=0.3$, being $\alpha$ the lapse function. The cores of the merging NSs are not significantly shocked during merger, so the central part of the remnant maintains a relatively low specific entropy of $s \lesssim 2 k_{\mathrm{B}}$. Material squeezed out of the collisional interface between the NSs forms the bulk of the disk and has entropy of a few $k_{\mathrm{B}}$. In the cases in which black hole formation occurs, the disk entropy is slightly higher (by a couple of $k_{\mathrm{B}}$ ) than in the cases in which a massive NS is still present at the center. 

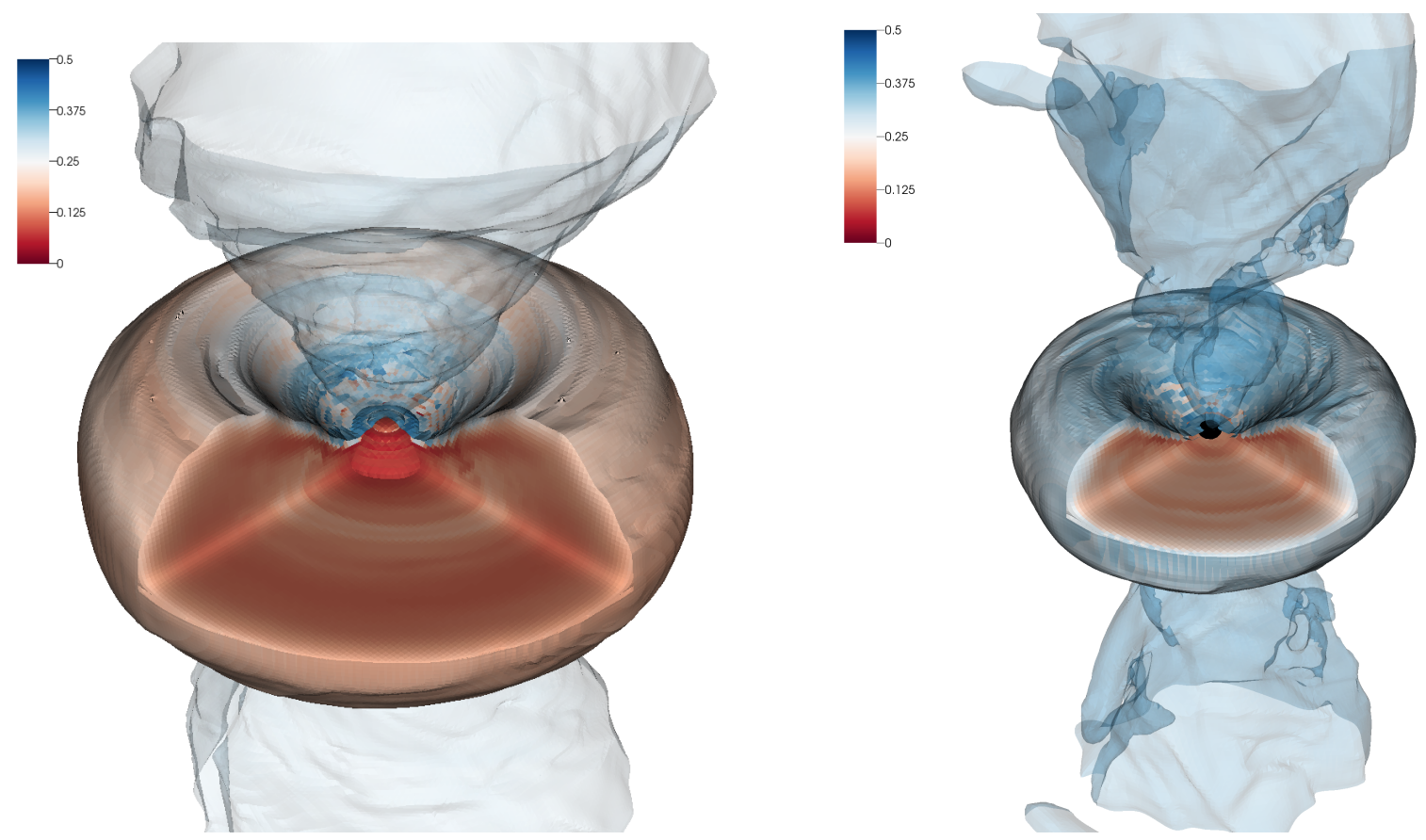

Fig. 13. Visualization of the electron fraction of the merger remnant. Left panel: DD2_M136136 at $t=34.2 \mathrm{~ms}$ after the merger. Right panel: SFHo_M135135 at $t=17.2 \mathrm{~ms}$ after the merger. Both image have the same spatial scale as in Fig. 11 and show the data in a box of size $\sim 750 \mathrm{~km}$. The electron fraction is used to color the $10^{7} \mathrm{~g} \mathrm{~cm}^{-3}$ (semi-transparent), and the $10^{11} \mathrm{~g} \mathrm{~cm}{ }^{-3}$ density iso-surfaces. We also show the electron fraction on the $\rho=10^{13} \mathrm{~g} \mathrm{~cm}^{-3}$ iso-surface for the DD2_M13641364 model. The black surface denotes the approximate location of the black hole horizon, which we identify as $\alpha=0.3$, being $\alpha$ the lapse function. The accretion disks are fairly neutron rich in their bulk, irrespective of the remnant type (massive NS or black hole). The accretion disk coronae are irradiated by neutrinos and are less neutron rich. 